\title{
Bifurcation analysis of nonlinear Hamiltonian dynamics in the Fermilab Integrable Optics Test Accelerator
}

\author{
Chad E. Mitchellø, ${ }^{*}$ Robert D. Ryne, and Kilean Hwang® \\ Lawrence Berkeley National Laboratory, Berkeley, California 94720, USA
}

(Received 11 March 2020; accepted 27 May 2020; published 15 June 2020)

\begin{abstract}
The Integrable Optics Test Accelerator (IOTA) is a novel storage ring at Fermi National Accelerator Laboratory designed (in part) to investigate the dynamics of beams in the presence of highly nonlinear transverse focusing fields that generate integrable single-particle motion with a large spread in the intrinsic betatron tunes. We describe how contemporary geometrical methods from the theory of integrable Hamiltonian systems may be used to locate all critical separatrixlike structures in the $4 \mathrm{D}$ transverse phase space, and to construct a complete analysis of the dynamical bifurcations of the system. Application of these techniques results in a global picture of the nominal on-energy transverse dynamics, revealing a rich diversity of accessible dynamical behavior. Similar techniques may be applied to future facilities that exploit the concept of nonlinear integrable optics.
\end{abstract}

DOI: 10.1103/PhysRevAccelBeams.23.064002

\section{INTRODUCTION}

The Integrable Optics Test Accelerator (IOTA) is a storage ring at Fermi National Accelerator Laboratory designed (in part) to investigate the dynamics of beams in the presence of highly nonlinear transverse focusing fields that generate integrable single-particle motion with large intrinsic betatron tune spreads $\left(\Delta \nu_{x, y}>0.25\right)$ [1,2]. A primary operational goal is to determine the degree to which the decoherence of transverse oscillations may be used to mitigate the development of instabilities [3] and core-halo resonances at high space charge intensity [4,5]. In comparison with nonlinear damping techniques using octupoles [6,7], integrability of the nonlinear Hamiltonian motion is enforced by design to improve the transverse dynamic aperture and beam confinement $[1,8]$.

The nominal transverse dynamics is described by an integrable Hamiltonian in two degrees of freedom containing a single dimensionless parameter (the nonlinear insert strength $\tau$ ). In this paper, we apply contemporary geometric techniques developed for studying the singularities of integrable Hamiltonian systems [9-11] to obtain a global picture of the qualitative dynamical behavior of this system, including the fixed points, stable and unstable periodic orbits, and phase space separatrices. The word "bifurcation" of the title is used in two distinct ways: (1) to refer to

\section{"ChadMitchell@lbl.gov}

Published by the American Physical Society under the terms of the Creative Commons Attribution 4.0 International license. Further distribution of this work must maintain attribution to the author(s) and the published article's title, journal citation, and DOI. the bifurcation diagram of an integrable Hamiltonian system, which provides a visual representation of those values of the invariants of motion where the topology of the invariant level set changes, and (2) to refer to a sudden global change in the system dynamics as one or more parameters in the Hamiltonian is continuously varied (in this case, the parameter $\tau$ ). Both meanings are intended here, and their relationship is considered.

Analysis of integrable Hamiltonian systems is often performed using one or more sets of action-angle phase space coordinates. In general, such action-angle coordinates are defined only locally, and break down near critical phase space structures (e.g., the separatrix of the nonlinear pendulum). In addition, canonical transformations to such coordinates are difficult to obtain in explicit form in even the simplest systems. The techniques described here do not require the use of action-angle coordinates, and are sufficiently general to be applied to future machine designs [12] described by an autonomous Hamiltonian, in which a second invariant is analytically known. Related techniques have recently had a major impact in molecular spectroscopy [13-15], and have been applied to a number of systems of physical interest $[16,17]$.

The layout of this paper is as follows. In Sec. II, we describe the IOTA nonlinear magnetic insert and the Hamiltonian of the associated dynamical system. In Sec. III, we describe the critical points and the bifurcation diagram of a general integrable Hamiltonian system, and we apply these concepts to IOTA. Section IV discusses the classification of critical points in detail. In Sec. V, we demonstrate how this information may be used to classify the orbits of IOTA at nominal operation. In Sec. VI, we extend this analysis to describe the dependence of these 
dynamical properties on the nonlinear insert strength parameter, and we consider bifurcations of the dynamical fixed points. The paper ends with a Conclusion, which includes a summary of key results. There are five Appendixes.

\section{HAMILTONIAN DESCRIPTION OF THE IOTA RING}

The IOTA ring design (for operation of the integrable optics experiment) consists of a "bare" linear lattice, with an available $1.8 \mathrm{~m}$ long dispersion-free drift space for the introduction of a magnetic insert [18] with highly nonlinear transverse fields to provide strong betatron detuning. Detailed analysis of the IOTA bare lattice optics may be found elsewhere [2]. In this section, we provide a brief summary of the Hamiltonian formalism describing the ideal nonlinear operation $[1,19]$.

\section{A. The IOTA nonlinear magnetic insert}

The ideal 2D magnetic field within the nonlinear insert is given at each longitudinal location $s$ by $\vec{B}=B_{x} \hat{x}+B_{y} \hat{y}$ satisfying $\partial_{x} B_{x}+\partial_{y} B_{y}=0$ and $\partial_{x} B_{y}-\partial_{y} B_{x}=0$. This field is most easily expressed in terms of either a magnetic vector potential $\vec{A}=A_{s} \hat{s}$ or a magnetic scalar potential $\psi$ satisfying $\vec{B}=\nabla_{\perp} \times \vec{A}=-\nabla_{\perp} \psi$ at each $s$, where the two potentials are given by the real and imaginary parts of the function [19]:

$$
F(z)=\left(\frac{z}{\sqrt{1-z^{2}}}\right) \arcsin (z)
$$

in terms of the dimensionless quantities:

$$
\tilde{t} F=\frac{A_{s}+i \psi}{B \rho}, \quad z=\frac{X+i Y}{c \sqrt{\beta(s)}}, \quad \tilde{t}=\frac{\tau c^{2}}{\beta(s)} .
$$

Here $\tau$ is a dimensionless parameter characterizing the strength of the magnet, $c \neq 0\left[\mathrm{~m}^{1 / 2}\right]$ characterizes the length scale of the potentials in the transverse plane, $B \rho$ is the magnetic rigidity, and $\beta=\beta_{x}=\beta_{y}$ is the betatron amplitude across the drift space in the bare lattice ("insert drift") that will contain the magnet, which is given explicitly by:

$$
\frac{\beta(s)}{\beta^{*}}=1+\left(\frac{2 s}{L}\right)^{2} \tan ^{2} \pi \mu_{0}, \quad \text { for }-\frac{L}{2} \leq s \leq \frac{L}{2} .
$$

In (3), $L$ denotes the length of the nonlinear insert, $0 \leq$ $\mu_{0}<1 / 2$ denotes the bare lattice tune advance across the insert drift, and $\beta^{*}$ denotes the betatron amplitude at the midpoint of the insert drift, given in terms of the parameters $L$ and $\mu_{0}$ by:

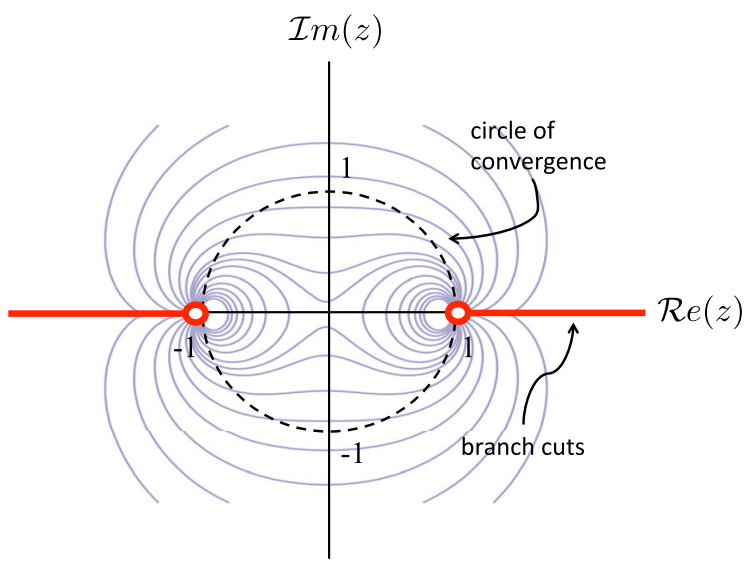

FIG. 1. Domain of analyticity of the complex function $F$, which defines the vector potential of the nonlinear insert in the transverse plane. The curves in blue denote magnetic field lines. The dashed circle denotes the circle of convergence of the multipole series. Singularities occur at the points $z= \pm 1$.

$$
\beta^{*}=\frac{L}{2} \cot \pi \mu_{0} .
$$

The function $F$ is analytic in the domain of the complex plane shown in Fig. 1, which excludes the two branch cuts (shown in red) and the two singular points $z= \pm 1$. The curves in blue denote the corresponding transverse magnetic field lines, which coincide with the contours of $A_{s}$. The magnetic multipole series can be obtained directly from the power series for (1):

$$
F(z)=\sum_{n=1}^{\infty} \frac{2^{2 n-1} n !(n-1) !}{(2 n) !} z^{2 n}, \quad|z|<1 .
$$

In practice, however, one is often interested in the dynamics well outside the radius of convergence of the series (5).

\section{B. Construction of the integrable Hamiltonian}

Since $\vec{A}_{\perp}=0$ in this model, the single-particle Hamiltonian within the nonlinear magnetic insert takes the following form, using the longitudinal coordinate $s$ as the independent variable [20]:

$$
H=-\sqrt{1-\frac{2 P_{t}}{\beta_{0}}+P_{t}^{2}-|\vec{P}|^{2}}-\mathcal{A}_{s}-\frac{1}{\beta_{0}} P_{t},
$$

where the transverse momenta $\vec{P}$ are normalized by the design momentum $p^{0}=m c \beta_{0} \gamma_{0}$, the longitudinal variables are $T=c \Delta t$ and $P_{t}=-\Delta \gamma /\left(\beta_{0} \gamma_{0}\right)$, and $\mathcal{A}_{s}=A_{s} / B \rho$. In the paraxial approximation $P_{x}, P_{y} \ll 1$, the Hamiltonian for an on-energy particle $\left(P_{t}=0\right)$ within the nonlinear magnetic insert takes the form:

$$
H_{\perp}\left(X, P_{x}, Y, P_{y} ; s\right)=\frac{1}{2}\left(P_{x}^{2}+P_{y}^{2}\right)-\mathcal{A}_{s}(X, Y, s) .
$$




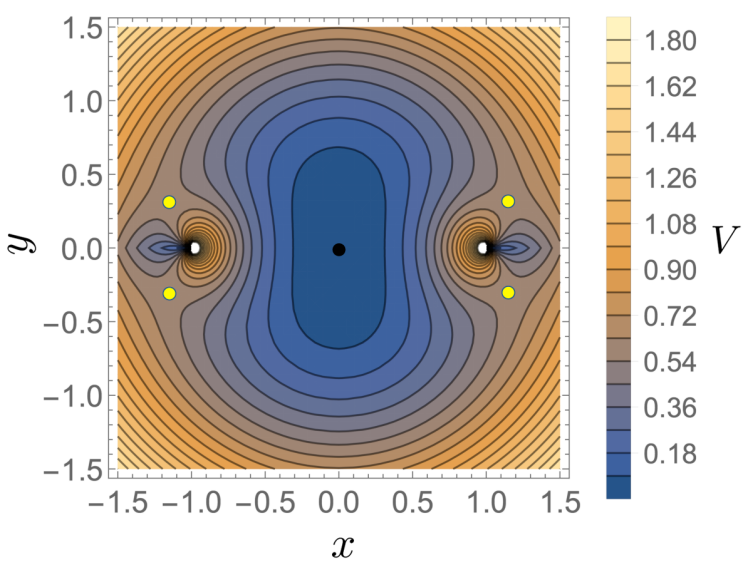

FIG. 2. Contours of the nonlinear potential (10) for a typical insert strength $\tau=-0.4$. Singularities occur at the points $(x, y)=( \pm 1,0)$. The potential is continuous away from these points and real-analytic away from the branch cuts $(|x| \geq 1$, $y=0$ ). Symmetry exists under reflections $x \mapsto-x$ and $y \mapsto-y$. Note the presence of one minimum (black dot) and 4 saddle points (yellow dots).

After making an s-dependent transformation to the dimensionless phase space variables [1]:

$$
\begin{array}{ll}
x=\left(\frac{1}{c \sqrt{\beta}}\right) X, & p_{x}=\left(\frac{\alpha}{c \sqrt{\beta}}\right) X+\left(\frac{\sqrt{\beta}}{c}\right) P_{x}, \\
y=\left(\frac{1}{c \sqrt{\beta}}\right) Y, & p_{y}=\left(\frac{\alpha}{c \sqrt{\beta}}\right) Y+\left(\frac{\sqrt{\beta}}{c}\right) P_{y},
\end{array}
$$

where $\alpha(s)=-\beta^{\prime}(s) / 2$, and using the bare lattice betatron phase advance $\psi\left(\right.$ defined by $\left.\psi^{\prime}=1 / \beta\right)$ as the independent variable, the Hamiltonian takes the autonomous form:

$$
H\left(x, p_{x}, y, p_{y}\right)=\frac{1}{2}\left(p_{x}^{2}+p_{y}^{2}\right)+V(x, y),
$$

where the potential function $V$ is given by $(z=x+i y)$ :

$$
\begin{aligned}
& V(x, y)=\frac{1}{2}\left(x^{2}+y^{2}\right)-\tau U(x, y), \\
& U(x, y)=\mathcal{R} e\left(\frac{z}{\sqrt{1-z^{2}}} \arcsin (z)\right) .
\end{aligned}
$$

Note that (10) is characterized by the single dimensionless parameter $\tau$ (nonlinear insert strength). The nonlinear potential $V$ is shown in Fig. 2 for a typical value of $\tau$. In the remainder of this paper, the terms "singularity" and "singular point" refer exclusively to the spatial points $(x, y)=( \pm 1,0)$ where the potential $V$ diverges.

The bare lattice optics in IOTA (from the exit of the nonlinear insert to its entrance) is designed to provide equal linear focusing in the horizontal and vertical planes, with corresponding phase advance given by $2 \pi k$, for integer $k$ [21]. As a consequence, the transfer map between passes through the magnetic insert is described in coordinates (8) by the identity map, and (9) is sufficient to describe the dynamics of the IOTA ring.

The Hamiltonian (9) admits an exactly-known integral of motion (invariant) of the form:

$$
\begin{aligned}
I\left(x, p_{x}, y, p_{y}\right) & =\left(x p_{y}-y p_{x}\right)^{2}+p_{x}^{2}+x^{2}-\tau W(x, y), \\
W(x, y) & =\mathcal{R} e\left(\frac{z+\bar{z}}{\sqrt{1-z^{2}}} \arcsin (z)\right),
\end{aligned}
$$

where $\bar{z}$ denotes the complex conjugate of $z=x+i y$. It may be directly verified that $\{H, I\}=0$, where $\{\cdot, \cdot\}$ denotes the classical Poisson bracket [19]. In addition, we will see that $\nabla H$ and $\nabla I$ are linearly independent everywhere except on a set of zero measure (phase space volume), so that the pair $(H, I)$ forms an integrable Hamiltonian system [22-24]. The remainder of this paper concerns the dynamics of the system described by (9)-(11).

\section{CRITICAL POINTS AND THE BIFURCATION DIAGRAM}

\section{A. The momentum mapping and its critical points}

Suppose $H$ is an integrable Hamiltonian for an $n$ degreeof-freedom system on a phase space $M$, and let $H=$ $f_{1}, \ldots, f_{n}$ denote its $n$ invariants of motion. The momentum mapping [9] is the smooth function $\mathcal{F}: M \rightarrow \mathbb{R}^{n}$ given by:

$$
\mathcal{F}(p)=\left(f_{1}(p), \ldots, f_{n}(p)\right), \quad p \in M .
$$

At any point $p \in M$, the Jacobian $D \mathcal{F}_{p}$ of (12) using phase space coordinates $\left(\zeta_{1}, \zeta_{2}, \ldots, \zeta_{2 n}\right)$ is the $n \times 2 n$ matrix given by:

$\left[D \mathcal{F}_{p}\right]_{j k}=\frac{\partial f_{j}}{\partial \zeta_{k}} \quad(j=1, \ldots, n, k=1, \ldots, 2 n)$.

A point $p$ in $M$ is a critical point of the momentum mapping $\mathcal{F}$ if

$$
\operatorname{rank}\left(D \mathcal{F}_{p}\right)<n
$$

Let $K$ denote the set of critical points. If $p \in K$, its image $\mathcal{F}(p)$ in $\mathbb{R}^{n}$ is called a critical value. The set of all critical values $\Sigma=\mathcal{F}(K)$ is called the bifurcation diagram of $H$.

The following observations illustrate the importance of these concepts.

(i) Since each $f_{1}, \ldots, f_{n}$ is invariant under the Hamiltonian flow, each orbit is confined to lie on a level set of $\mathcal{F}$. These invariant level sets partition the entire phase space $M$.

(ii) A regular level set of $\mathcal{F}$ is one that contains no critical points. Its compact connected components are invariant $n$-dimensional tori, as described by the Liouville-Arnold theorem [22].

(iii) A critical level set of $\mathcal{F}$ is one corresponding to a critical value in $\Sigma$. The critical level sets include 
exceptional lower-dimensional tori, fixed points, periodic orbits, and their stable and unstable manifolds.

(iv) The bifurcation diagram $\Sigma$ contains those values $\left(f_{1}, \ldots, f_{n}\right) \in \mathbb{R}^{n}$ where the level sets of $\mathcal{F}$ undergo topological (qualitative) change as the invariants $f_{j}$ are varied.

The momentum mapping in the case of IOTA is given explicitly by:

$\mathcal{F}\left(x, p_{x}, y, p_{y}\right)=\left(H\left(x, p_{x}, y, p_{y}\right), I\left(x, p_{x}, y, p_{y}\right)\right)$,

where $H$ and $I$ are the two invariants of motion given in (9)-(11). To search for a critical point $p=\left(x, p_{x}, y, p_{y}\right)$ of (15), one may check any of the following four equivalent conditions:

(1) $\operatorname{rank}\left(D \mathcal{F}_{p}\right)<2$,

(2) $\operatorname{det}\left(D \mathcal{F}_{p}\right)\left(D \mathcal{F}_{p}\right)^{T}=0$,

(3) $\nabla H(p)$ and $\nabla I(p)$ are linearly dependent,

(4) $d H \wedge d I=0$ at $p$.

Finding the set of critical points using (16) requires searching for the simultaneous zeros of one or more functions of four variables. For example, applying condition (16d), which is expressed using exterior differential forms, results in a set of six functions to be zeroed. In Appendix A, we provide additional details and summarize the main families of critical points obtained using this method.

A detailed classification of the critical points is provided in Sec. IV. We remark that the theory [9] assumes that $f_{j}$ $(j=1, \ldots, n)$ are smooth, while the two functions $H$ and $I$ have vertical derivatives that are discontinuous across the two branch cuts. The latter fact has a few (minor) consequences that will play a role in later sections.

\section{B. Bifurcation diagram of IOTA}

Taking the image under $\mathcal{F}$ of the set $K$ of critical points obtained using (16) yields the bifurcation diagram $\Sigma=\mathcal{F}(K)$. In general, the points on the bifurcation diagram must be obtained numerically. In the special case of the Hamiltonian (9)-(10), an explicit parametrization is possible, as described in Appendix C. The result consists of the union of the following four parametrized curves in the $(H, I)$ plane:

$$
\begin{aligned}
H_{A} & =\frac{1}{2}\left(-1+2 \xi^{2}+\tau+\frac{\left(-1+2 \xi^{2}\right) \tau \operatorname{arccosh} \xi}{\xi \sqrt{\xi^{2}-1}}\right), \\
I_{A} & =\xi\left(\xi^{3}+\xi \tau+\frac{\tau \operatorname{arccosh} \xi}{\sqrt{\xi^{2}-1}}\right), \quad \xi \geq \xi_{\min }
\end{aligned}
$$

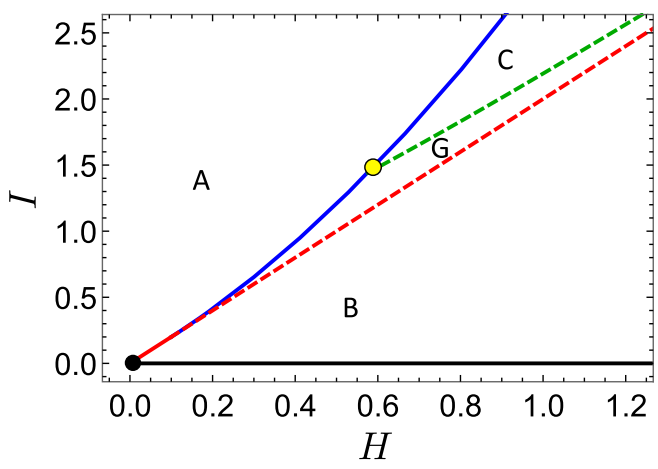

FIG. 3. Bifurcation diagram $\Sigma$ for the integrable Hamiltonian system of IOTA (9)-(11) at the nominal operating value $\tau=-0.4$, separating the $(H, I)$ plane into four regions with distinct dynamical behavior. Curves in blue, green, red, and black correspond to the four curves in (17), respectively. Significance of the solid-dashed distinction and the large dots is explained in Sec. IV C.

$$
\begin{aligned}
& H_{B}=\frac{1}{2}\left(-1+2 \eta^{2}+\tau+\frac{\left(1-2 \eta^{2}\right) \tau \arcsin \eta}{\eta \sqrt{1-\eta^{2}}}\right), \\
& I_{B}=\eta\left(\eta^{3}+\eta \tau-\frac{\tau \arcsin \eta}{\sqrt{1-\eta^{2}}}\right), \quad \eta_{\min } \leq \eta \leq 1 \\
& H_{C}=s, \quad I_{C}=2 s, \quad s \geq s_{\min } \\
& H_{D}=t, \quad I_{D}=0, \quad t \geq t_{\min }
\end{aligned}
$$
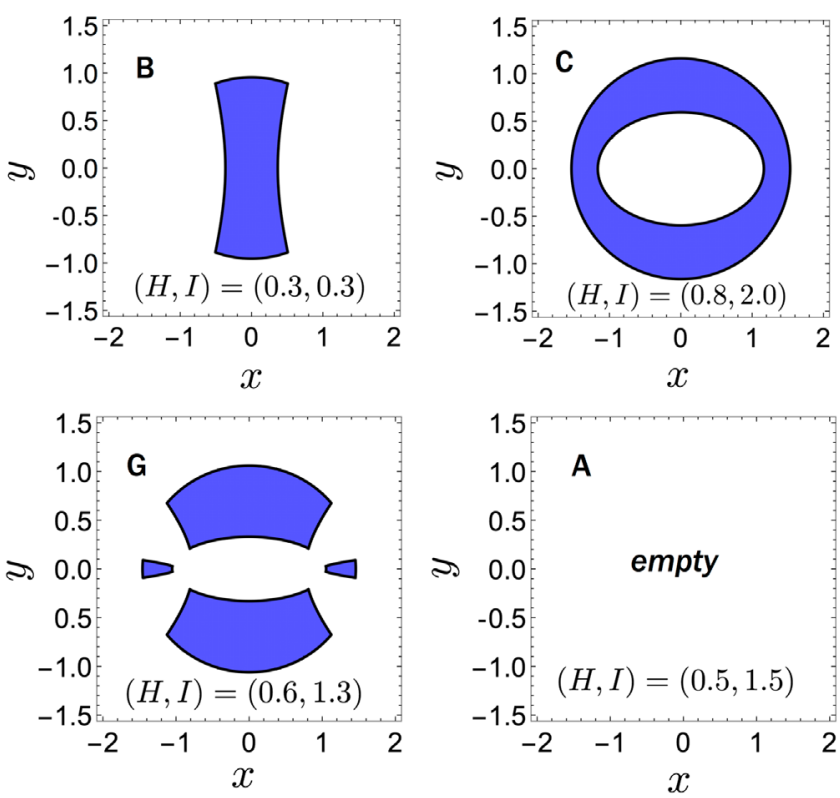

FIG. 4. Projections of level sets from each of the four regions shown in Fig. 3 ( $\tau=-0.4)$. A non-resonant orbit on such a level set densely fills one of its connected components. (B) One connected component, not intersecting the branch cuts. (C) One connected component, intersecting the branch cuts (hole visible). (G) Four connected components. (A) Empty. 
The values $\left(\xi_{\min }, \eta_{\min }, s_{\min }, t_{\min }\right)$ depend on the nonlinear insert strength $\tau$. For a value in the nominal range $-1 / 2<$ $\tau<0$, one has $\xi_{\min }=1, s_{\min }=t_{\min }=0$, and $\eta_{\min }=\bar{\eta}$, where $(\bar{\xi}, \bar{\eta})$ is the unique solution of $H_{A}(\bar{\xi})=H_{B}(\bar{\eta})$, $I_{A}(\bar{\xi})=I_{B}(\bar{\eta})$.

Figure 3 illustrates the bifurcation diagram for the case $\tau=-0.4$. The curves partition the plane into four regions. All values of $(H, I)$ appearing in the interior of a given region generate regular level sets $\mathcal{F}^{-1}(\{(H, I)\})$ of the same topological type. Each of these regular level sets is a 2-dimensional surface embedded in the 4-dimensional phase space $M$, which is readily illustrated via its projection into the $(x, y)$ plane. (See Appendix B for details.) We find a total of 7 distinct types of regular level sets, indicated by the codes A-G. Only four of these are accessible when $\tau=-0.4$. They are shown in Fig. 4. Values of $(H, I)$ that lie on the curves in Fig. 3 generate critical level sets that correspond to transitions between these four cases. These critical level sets form the skeleton of the global dynamics, and are treated in subsequent sections.

\section{CLASSIFICATION OF CRITICAL POINTS}

In (14), the integer $0 \leq \operatorname{rank}\left(D \mathcal{F}_{p}\right)<n$ is called the rank of the critical point. Consider the system (9)-(11), with invariants $f_{1}=H$ and $f_{2}=I$. The rank of a critical point $p \in K$ may also be expressed as:

$$
\operatorname{rank}\left(D \mathcal{F}_{p}\right)=\operatorname{dim}(\operatorname{Span}\{\nabla H(p), \nabla I(p)\}) .
$$

Critical points of rank 0 and of rank 1 are discussed below. It is significant that the dynamical stability of these critical points can be analyzed using only knowledge of the two invariants of motion $H$ and $I$. For a comprehensive approach to the classification of critical points in a general integrable Hamiltonian system, see [9-11]. For the system (9)-(11), we use the simplified classification scheme described below, which is sufficient for our purposes.

\section{A. Fixed points}

Let $p \in M$ be a rank- 0 critical point of the momentum mapping $\mathcal{F}$. From (18), it follows that $\nabla H(p)=0$. Since the Hamiltonian equations of motion take the form (Appendix D):

$$
\dot{\zeta}=J \nabla H(\zeta)
$$

it follows that $p$ is an equilibrium point (fixed point) of the Hamiltonian flow. Conversely, it is possible to prove using (9)-(11) that $\nabla H(p)=0$ implies $\nabla I(p)=0$, and we conclude from (18) that the set of rank-0 critical points coincides with the set of dynamical fixed points.

As described in Appendix D, the dynamical stability of a fixed point is determined by the eigenvalues of the $4 \times 4$ matrix $J S$, where $S$ is the Hessian matrix of $H$ at $p$ :
$S_{i j}=\left(\operatorname{Hess}_{p} H\right)_{i j}=\frac{\partial^{2} H}{\partial \zeta_{i} \partial \zeta_{j}}(p), \quad(i, j=1, \ldots, 4)$.

For a Hamiltonian of the form (9), we see that $p=$ $\left(x, p_{x}, y, p_{y}\right)$ is a fixed point if and only if:

$$
p_{x}=p_{y}=0, \quad \nabla V(x, y)=0 .
$$

The analysis of stability is then reduced to studying the eigenvalues of the $2 \times 2$ Hessian matrix of the potential $V$ (Appendix D).

It is convenient to express the gradient of the potential $V$ of (10) in the form:

$$
\nabla V(x, y)=\left(x-\tau \mathcal{R} e F^{\prime}, y+\tau \mathcal{I} m F^{\prime}\right),
$$

where $F$ is given in (1), and the complex derivative $F^{\prime}$ is evaluated at the point $z=x+i y$. The fixed point condition (21) can then be expressed as the following equation in the complex plane:

$$
\bar{z}=\tau F^{\prime}(z), \quad z=x+i y .
$$

Likewise, the Hessian matrix of second derivatives of $V$ can be expressed as:

$$
\operatorname{Hess}(V)=\left(\begin{array}{cc}
1-\tau \mathcal{R} e F^{\prime \prime} & \tau \mathcal{I} m F^{\prime \prime} \\
\tau \mathcal{I} m F^{\prime \prime} & 1+\tau \mathcal{R} e F^{\prime \prime}
\end{array}\right),
$$

where $F^{\prime \prime}$ is evaluated at the point $z=x+i y$. Stability at a fixed point $z_{c} \in \mathbb{C}$ satisfying (23) is determined by the two eigenvalues of (24), given explicitly by:

$$
\lambda_{ \pm}=1 \pm \tau\left|F^{\prime \prime}\left(z_{c}\right)\right|, \quad z_{c}=x_{c}+i y_{c} .
$$

Note that one of the values in (25) is always positive, and the sign of the second value coincides with the sign of the determinant $\lambda_{+} \lambda_{-}$.

Given a fixed point $p \in M$, we say that $p$ is stable, unstable, or degenerate if $\operatorname{det}\left(\operatorname{Hess}_{p}(V)\right)>0$, $\operatorname{det}\left(\operatorname{Hess}_{p}(V)\right)<0$, or $\operatorname{det}\left(\operatorname{Hess}_{p}(V)\right)=0$, respectively. We note that this classification is coordinate-independent. (That is, any choice of smooth coordinates near $p$ may be used to evaluate the Hessian matrix.) Examples of stable and unstable fixed points may be seen in Fig. 2 for the nominal insert strength $\tau=-0.4$, given by the black and yellow dots, respectively.

\section{B. Periodic orbits}

Next, let $p \in M$ be a rank-1 critical point of the momentum mapping $\mathcal{F}$. It follows from (18) that the gradients of $H$ and $I$ are parallel at $p$. Informally, the level set containing $p$ looks locally 1-dimensional at $p$. (For example, the level set may contain a "cusp" along a curve containing $p$.) If the level set is compact, this typically 
implies that $p$ lies on a periodic orbit. We refer to such orbits as critical periodic orbits, to distinguish them from periodic orbits that lie on those regular level sets with resonant characteristic frequencies.

To study such a periodic orbit, let $S$ be a 2D Poincaré section transverse to the periodic orbit of $p$ within the 3D isoenergy surface. Explicitly, define a set of the form:

$$
S=\left\{\zeta \in M: H(\zeta)=H_{0}, G(\zeta)=G_{0}\right\},
$$

where $H_{0}=H(p), G_{0}=G(p)$, and $G$ is any smooth function on $M$ with:

$$
\left.\{H, G\}\right|_{p} \neq 0 .
$$

Then $S$ defines a smooth 2D surface in a neighborhood of $p$, and $p$ is a fixed point of the Poincare return map on this surface.

Consider the restriction of the invariant function $I$ to the surface $S$, denoted by $\left.I\right|_{S}$. Since $\operatorname{rank}\left(D \mathcal{F}_{p}\right)=1$, it follows that the function $\left.I\right|_{S}$ has vanishing derivatives at $p$, in the sense that $D\left(\left.I\right|_{S}\right)_{p}=0$. We say that $p$ is stable, unstable, or degenerate if $\operatorname{det}\left(\operatorname{Hess}_{p}\left(\left.I\right|_{S}\right)\right)>0, \operatorname{det}\left(\operatorname{Hess}_{p}\left(\left.I\right|_{S}\right)\right)<0$, or $\operatorname{det}\left(\operatorname{Hess}_{p}\left(\left.I\right|_{S}\right)\right)=0$, respectively. It can be shown that this classification is independent of the choice of $G$ satisfying (27) in the definition of $S$, and it is also independent of the set of coordinates on $S$ used to evaluate this $2 \times 2$ Hessian matrix.

This is best illustrated by an example. For the system (9)-(11), consider a point $p \in M$ of the form:

$$
\left(x, p_{x}, y, p_{y}\right)=(0, a, 0,0), \quad a>0 .
$$

We see from Appendix A (case 4) that any such point is a critical point of $\mathcal{F}$. Since $p_{x}=a$ is nonzero, this critical point has rank 1 and energy $H_{0}=a^{2} / 2$. It is not difficult to see (using the symmetry of $V$ ) that $p$ lies on a periodic orbit confined to the $\left(x, p_{x}\right)$ plane. In (26), take $G\left(x, p_{x}, y, p_{y}\right)=$ $x$ and $G_{0}=0$. The criterion (27) is satisfied since:

$$
\left.\{H, G\}\right|_{p}=\left.\{H, x\}\right|_{p}=-a \neq 0 .
$$

Then $S$ in (26) defines a smooth 2D surface near $p$, namely the Poincare section $H=H_{0}, x=0$, which intersects the orbit transversely. On this surface, we have:

$$
p_{x}=g\left(y, p_{y}\right), \quad g\left(y, p_{y}\right)=\sqrt{2\left(H_{0}-V(0, y)\right)-p_{y}^{2}} .
$$

The restriction of $I$ to this surface is then given [in the coordinates $\left.\left(y, p_{y}\right)\right]$ by:

$$
\left.I\right|_{S}\left(y, p_{y}\right)=I\left(0, g\left(y, p_{y}\right), y, p_{y}\right) .
$$

Figure 5 shows contours of the function $\left.I\right|_{S}$ for two values of $H_{0}$ at the nominal insert strength $\tau=-0.4$. The periodic orbit of the point (28) intersects $S$ at the origin, and it is stable

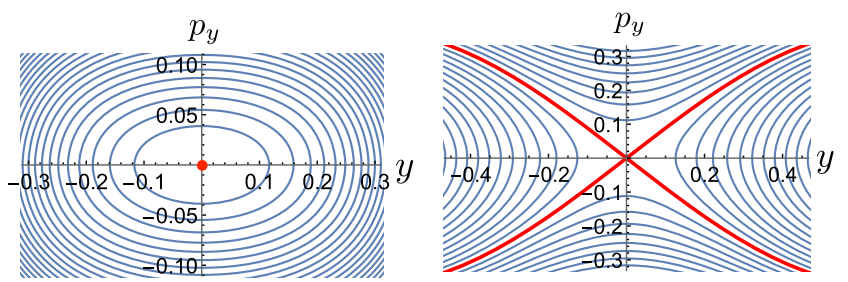

FIG. 5. Stable and unstable critical periodic orbits $(\tau=-0.4)$. Contours of the function $\left.I\right|_{S}$ are shown on the surface $S$ defined by $H=H_{0}, x=0$ near the critical point (28). The periodic orbit of $p$ pierces the surface $S$ at the origin. (Left) The case $H_{0}=0.05$, for which $p$ is stable. (Right) The case $H_{0}=0.5$, for which $p$ is unstable. The visible portion of the level set containing $p$ is shown in red.

when $H_{0}<0.1$, unstable when $H_{0}>0.1$, and degenerate when $H_{0}=0.1$.

A summary of the classification scheme described in Secs. IVA and IV B is provided in Table I, where a connection is made with the mathematical literature [11].

\section{Interpretation of the bifurcation diagram}

The bifurcation diagram $\Sigma$ encodes valuable information about the global structure of the integrable Hamiltonian system [9-11]. For a system with two degrees of freedom, each point on a well-behaved curve in $\Sigma$ corresponds to a level set containing a nondegenerate rank- 1 critical point ([9], Prop. 1.16). On the other hand, a "cusp" or intersection point in the bifurcation diagram generally indicates the presence of a degenerate rank- 1 critical point or a rank-0 critical point (fixed point). For the bifurcation diagram shown in Fig. 3, information regarding the classification of critical points is indicated as follows:

(i) A black dot indicates that the level set contains one or more fixed points, all of which are stable.

(ii) A yellow dot indicates that the level set contains one or more fixed points, at least one of which is unstable.

(iii) A solid line indicates that the level set contains one or more periodic orbits, all of which are stable.

TABLE I. Classification of a critical point $p$ by rank and stability. The terminology in parentheses is taken from [11].

\begin{tabular}{lc}
\hline \hline Type & Criterion \\
\hline Fixed point & $\operatorname{rank}\left(D \mathcal{F}_{p}\right)=0$ \\
stable (elliptic-elliptic) & $\operatorname{det}\left(\operatorname{Hess}_{p}(V)\right)>0$ \\
unstable (elliptic-hyperbolic) & $\operatorname{det}\left(\operatorname{Hess}_{p}(V)\right)<0$ \\
degenerate & $\operatorname{det}\left(\operatorname{Hess}_{p}(V)\right)=0$ \\
Periodic orbit & $\operatorname{rank}\left(D \mathcal{F}_{p}\right)=1$ \\
stable (transversally elliptic) & $\operatorname{det}\left(\operatorname{Hess}_{p}\left(\left.I\right|_{S}\right)\right)>0$ \\
unstable (transversally hyperbolic) & $\operatorname{det}\left(\operatorname{Hess}_{p}\left(\left.I\right|_{S}\right)\right)<0$ \\
degenerate & $\operatorname{det}\left(\operatorname{Hess}_{p}\left(\left.I\right|_{S}\right)\right)=0$ \\
\hline \hline
\end{tabular}


(iv) A dashed line indicates that the level set contains one or more periodic orbits, at least one of which is unstable.

The stability of each critical periodic orbit is determined by using an appropriate choice of Poincaré section, as described in the previous section. The results may be summarized using the Hessian determinant for a typical periodic orbit on the level sets obtained along each of the four parametrized curves given in (17):

$\operatorname{det}_{A}\left(\left.\operatorname{Hess} I\right|_{S}\right)=4\left(4 \xi^{4}+2 \xi^{2}(-2+\tau)-\tau-\frac{\tau \operatorname{arccosh} \xi}{\xi \sqrt{\xi^{2}-1}}\right)$,

$\operatorname{det}_{B}\left(\left.\operatorname{Hess} I\right|_{S}\right)=4\left(4 \eta^{4}+2 \eta^{2}(-2+\tau)-\tau+\frac{\tau \arcsin \eta}{\eta \sqrt{1-\eta^{2}}}\right)$,

$\operatorname{det}_{C}\left(\left.\operatorname{Hess} I\right|_{S}\right)=4-8 s+8 \tau$,

$\operatorname{det}_{D}\left(\left.\operatorname{Hess} I\right|_{S}\right)=4+8 t-8 \tau$.

Note that the choice of $S$ differs in each case. The above result is valid for any $\tau<0$. When $\tau \geq 0$, the stability results for case $\mathrm{C}$ are modified slightly.

\section{DYNAMICS AT NOMINAL INSERT STRENGTH}

In this section, we illustrate the relevance of the information contained in Figs. 3-4 to the dynamics of IOTA at the nominal insert strength $\tau=-0.4$. To do this, recall that the phase space $M$ is partitioned into level sets of the momentum mapping $\mathcal{F}$. The critical level sets play a central role, and their union is given by $\mathcal{F}^{-1}(\Sigma)$, where $\Sigma$ denotes the bifurcation diagram. We refer to $\mathcal{F}^{-1}(\Sigma)$ as the set of critical initial conditions in the phase space $M$. This set includes all the critical points of $\mathcal{F}$. In addition, it includes all points whose orbits may approach a critical point in the limit $t \rightarrow \infty$ or $t \rightarrow-\infty$ (stable and unstable manifolds). We will see that this set divides the phase space $M$ into regions with qualitatively distinct dynamical behaviors.

In order to visualize the set $\mathcal{F}^{-1}(\Sigma)$ in the $4 \mathrm{D}$ phase space $M$, we take its intersection with the plane defined by $p_{x}=p_{y}=0$. The resulting network of critical initial conditions in the $(x, y)$ plane is shown in Fig. 6. The various curves and points, as well as their colors and stability indicators (solid, dashed) correspond to those shown in Fig. 3 under the mapping $\mathcal{F}$. Note that there are five fixed points: one stable fixed point at the origin (black dot) and four unstable fixed points (yellow dots), which correspond also to the five points shown in Fig. 2. The letter codes correspond to the level set types shown in Fig. 4. Although only two level set types appear (B and G), we will see dynamical differences between the regions of type $\mathrm{G}$. The level set type $\mathrm{C}$ does not appear, as no orbit on such a level set contains a point with $p_{x}=p_{y}=0$.

\section{A. Orbits on regular level sets}

Consider the orbit of a particle with initial condition $p_{x}=p_{y}=0$ and coordinates $(x, y)$ in the transverse plane.

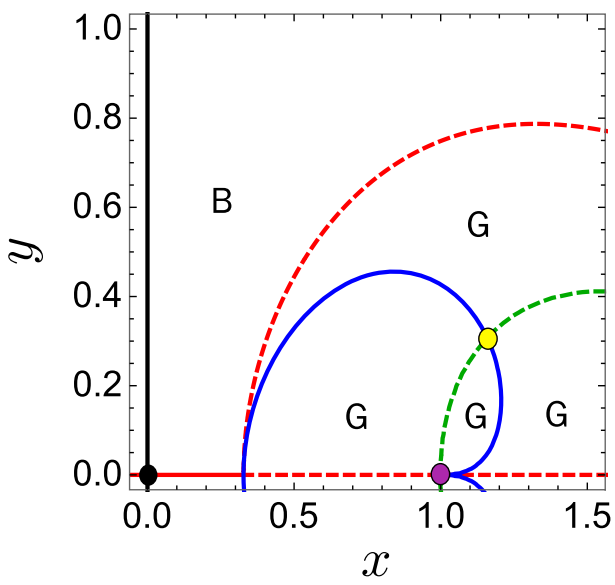

FIG. 6. Critical initial condition set $\mathcal{F}^{-1}(\Sigma)$, shown in the $(x, y)$ plane with $p_{x}=p_{y}=0$, for the nominal insert strength $\tau=-0.4$. Only one quadrant is shown. (Results are symmetric about the $x$ - and $y$-axes.) Colors and labels correspond to those found in Fig. 3. This set includes one stable fixed point (black dot, origin) and four unstable fixed points (yellow dots, one visible). The visible singular point is located at $(1,0)$ (purple), but is not considered part of the phase space $M$. A particle with its initial condition on one of the solid curves executes a stable periodic orbit.

If the point $(x, y)$ does not lie on one of the curves shown in Fig. 6, then the orbit lies on a regular level set of $\mathcal{F}$ consisting of one or more connected components (Liouville tori). (See Fig. 4.) Due to the continuity of the Hamiltonian flow, the orbit is confined exclusively to one of these connected components. Furthermore, if the characteristic frequencies are non-resonant, the orbit is dense on this component, and the projection of the level set into the $(x, y)$ plane provides a complete picture of the orbit geometry in this plane.

All such orbits can be classified as shown in Fig. 7. The three figures illustrate three shaded (gray) regions in the plane, and initial conditions in distinct regions have distinct orbit geometry. For reference, the network of critical initial conditions is shown in black. (The colors that appear in Fig. 6 have been removed to avoid visual clutter.) Note that the dashed red arc and the dashed green arc appearing in Fig. 6 define the boundaries of the shaded regions. Level sets of $\mathcal{F}$ corresponding to three distinct initial conditions are shown (blue), one from each region. In each case, the initial condition is located on one of the visible "corners" of the level set. (These corners correspond to the turning points where $p_{x}=p_{y}=0$ ).

For the initial condition $(x, y)=(0.3,0.6)$, the orbit executes motion surrounding the origin, filling densely a level set that is symmetric about the $x$ - and $y$-axes [Fig. 7(a)]. For the initial condition $(x, y)=(0.7,0.3)$, the orbit fills densely the upper component of the level set in Fig. 7(b), executing motion in the upper half-plane. In this case, the orbit effectively "bounces" off of the potential barrier formed by the presence of the singularities. 

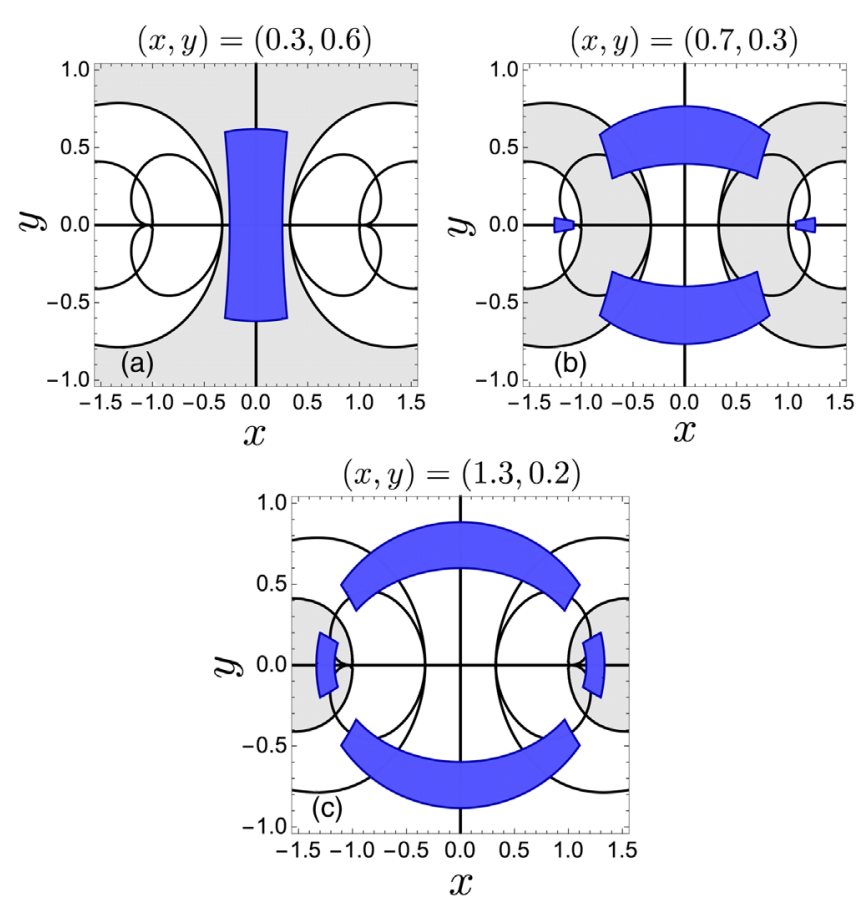

FIG. 7. Level sets (blue) for three distinct initial conditions with $p_{x}=p_{y}=0$, reflecting three distinct types of orbit geometry at the nominal insert strength $\tau=-0.4$. The set of critical initial conditions is shown in black. (a) The orbit is symmetric about the $x$ - and $y$-axes. (b) The orbit is confined to the upper half-plane. (c) The orbit is confined to the right half-plane.

(Likewise, the orbit of $(x, y)=(0.7,-0.3)$ is confined to the lower half-plane.) Finally, for the initial condition $(x, y)=(1.3,0.2)$, the orbit fills densely the small rightmost component of the level set in Fig. 7(c), executing motion in the right half-plane and crossing the branch cut. (Likewise, the orbit of $(x, y)=(-1.3,0.2)$ is confined to the left half-plane).

This behavior was verified by numerical tracking using a symplectic integrator. For example, Fig. 8 illustrates the orbit obtained for the initial condition $(x, y)=(0.7,0.3)$, together with the upper component of the level set shown in

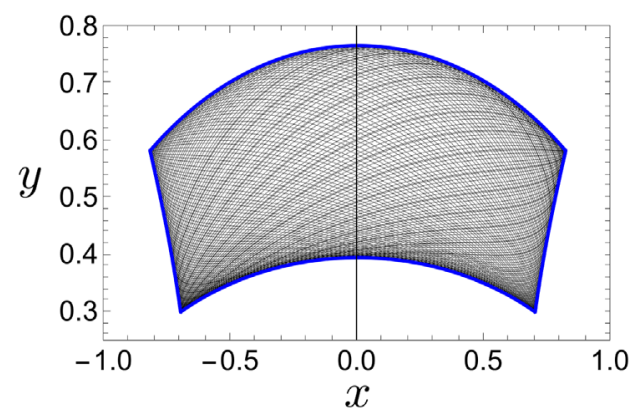

FIG. 8. The orbit obtained by numerical tracking of initial condition $p_{x}=p_{y}=0$ and $(x, y)=(0.7,0.3)$ for $\tau=-0.4$ is shown together with the upper component of the corresponding invariant level set, which is defined by $(H, I)=(0.39,0.86)$. The boundary of the level set appears in blue. Compare Fig. 7(b).
Fig. 7(b). The orbit comes arbitrarily close to each point within the blue boundary as $t \rightarrow \infty$. If the initial condition $(x, y)=(0.7,-0.3)$ is used, the orbit similarly fills the lower component in Fig. 7(b). Similar behavior occurs for the other orbits whose geometry is described in Fig. 7.

Note that we have considered orbits initialized from rest. However, there exist orbits with no turning points (points where $\left.p_{x}=p_{y}=0\right)$. An example is given by the initial condition $\left(x, p_{x}, y, p_{y}\right)=(0,1,1,0)$. One may verify that the corresponding point $(H, I)$ lies in the region C of Fig. 3, and the orbit fills densely a level set of type C (Fig. 4), encircling the origin.

\section{B. Orbits on critical level sets}

For an initial condition with $(x, y)$ located on one of the solid curves in Fig. 6, the corresponding level set of $\mathcal{F}$ is a $1 \mathrm{D}$ closed curve in the $4 \mathrm{D}$ phase space, indicating that motion occurs on a stable periodic orbit. For example, in Fig. 7(a), as the initial condition is moved toward the vertical (horizontal) axis, the projection of the level set shrinks to a vertical (horizontal) line segment. Similar behavior occurs for the shaded regions shown in Figs. 7(b)-(c). In these cases, as the black curve is approached the level set projection shrinks to the union of four segments of an ellipse, one of which coincides with the orbit.

The behavior of orbits for points on the dashed curves in Fig. 6 is more complex, as these are associated with separatrix-like structures in the phase space $M$. For a point on the dashed red arc, we obtain a level set that is intermediate between those shown in Figs. 7(a)-(b), with upper and lower components merged along a cusp with $y=0$. The dynamics near such a level set $(H, I)=$ $(0.5,1)$ is illustrated in Fig. 9 using a Poincaré section.

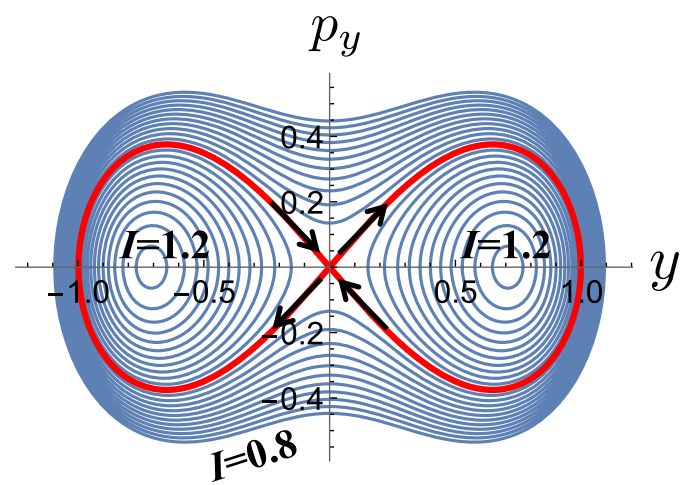

FIG. 9. Poincaré section illustrating the critical level set $(H, I)=(0.5,1)$ (red curve). Intersections of several level sets of $\mathcal{F}$ with the surface defined by $H=0.5, x=0$ are shown for $\tau=-0.4$. Curves in blue correspond to distinct values of $I$ ranging from $I=0.8$ to $I=1.2$. The critical level set contains an unstable periodic orbit, intersecting the surface at the origin. Black arrows denote the stable and unstable directions associated with this periodic orbit. In addition, two stable periodic orbits intersect the surface near $\left(y, p_{y}\right)=( \pm 0.7,0)$. 
(We examine the intersection of the level sets of $\mathcal{F}$ with the surface $S$ defined in (26) by taking $G=x, H_{0}=0.5$, $G_{0}=0$.) This level set contains (among others) points $\left(x, p_{x}, y, p_{y}\right)=(0.68,0,0,0)$ and $\left(x, p_{x}, y, p_{y}\right)=(0.95,0$, $0.74,0)$ that appear on the dashed red curves in Fig. 6. The first of these points yields an unstable periodic orbit that intersects the surface $S$ at the origin in Fig. 9. The second of these points yields an orbit whose intersections with the surface are confined to the red curve in Fig. 9. All such orbits on the red curve are homoclinic to the periodic orbit at the origin. Note that nearby orbits with $I<2 H_{0}$ cross the midplane $y=0$, while nearby orbits with $I>2 H_{0}$ do not. The latter behavior can also be seen by studying the line $H=H_{0}$ in Fig. 3 .

Similarly, as an initial condition is moved from the shaded region in Fig. 7(b) or (c) to the dashed green arc shown in Fig. 6, the upper and lower components of the level set merge with the right and left components of the level set. An initial condition exactly on the green arc yields an unstable periodic orbit that lies on a separatrix-like level set similar to that shown in Fig. 9.

Finally, the level set containing the 4 unstable fixed points (yellow dot in Fig. 6) is shown in Fig. 10 via its projections into the $(x, y)$ and $\left(x, p_{x}\right)$ planes. In addition to the fixed points themselves, the level set contains 8 heteroclinic orbits, each such orbit connecting one pair of unstable fixed points. This example also shows that a single level set may contain both rank- 0 and rank- 1 critical points.
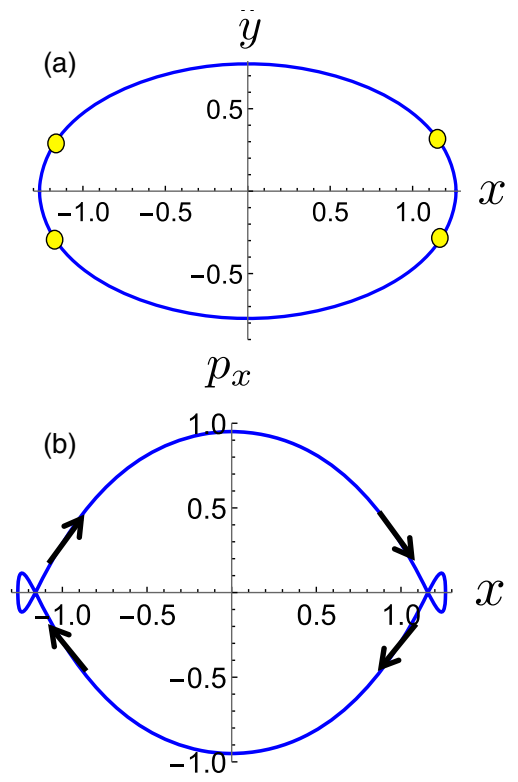

FIG. 10. Critical level set containing the four unstable fixed points for the case $\tau=-0.4$. (a) Projection into the $(x, y)$ plane. Fixed points are shown in yellow. (b) Projection into the $\left(x, p_{x}\right)$ plane. Fixed points are located at the two curve self-intersections. Black arrows denote stable and unstable directions near the fixed points. The projection of the level set into the $\left(y, p_{y}\right)$ plane has a similar structure.

\section{DEPENDENCE ON THE STRENGTH PARAMETER}

In this section, we study the dependence of the dynamics on the nonlinear insert strength $\tau$. In particular, we determine those values $\tau$ where bifurcation occurs.

\section{A. Bifurcation of fixed points}

Recall from (23) that fixed points of the Hamiltonian flow occur where $p_{x}=p_{y}=0$ and:

$$
\nabla V(x, y)=0 \Leftrightarrow \bar{z}=\tau F^{\prime}(z), \quad z=x+i y .
$$

It follows from (1) that $F^{\prime}(z)=0$ if and only if $z=0$, and the origin is therefore a fixed point for every $\tau$. To determine all other fixed points, we may solve for $\tau$ in (32) to obtain, after taking real and imaginary parts:

$$
\mathcal{R} e\left(\frac{\bar{z}}{F^{\prime}(z)}\right)=\tau, \quad \mathcal{I} m\left(\frac{\bar{z}}{F^{\prime}(z)}\right)=0 .
$$

Plotting the contour corresponding to the rightmost equation in (33) yields the set of black curves shown in Fig. 11. Contours of the leftmost equation in (33) are indicated by the dashed red lines, shown for several distinct values of $\tau$. For a given value of $\tau$, the fixed points appear at the intersection(s) of these curves. In this figure, one may follow the locations of the fixed points as $\tau$ is decreased from $\tau=1 / 2$ to $\tau=-9$.

We consider a fixed point bifurcation to occur when two fixed points split/merge from each other as $\tau$ is varied, or when a fixed point splits/merges from one of the singularities $( \pm 1,0)$. The former case is accompanied by a change in stability type. Thus, we determine all values of $\tau$ for which one or more degenerate fixed points is present. Recall that a degenerate fixed point occurs where (32) is satisfied and:

$$
\operatorname{det}(\operatorname{Hess} V(x, y))=0 \Leftrightarrow 1= \pm \tau\left|F^{\prime \prime}(z)\right| \text {. }
$$

These conditions are satisfied at exactly 3 triples $(x, y, \tau)$, namely those given by:

$$
(x, y, \tau)=\left(0,0, \pm \frac{1}{2}\right) \quad \text { or } \quad(x, y, \tau)=\left(0, y_{c}, \tau_{c}\right),
$$

where $y_{c} \approx 2.7$ and $\tau_{c} \approx-6.8$ are determined by solving:

$$
-3 y_{c} \sqrt{1+y_{c}^{2}}+\left(-1+2 y_{c}^{2}\right) \operatorname{asinh} y_{c}=0, \quad \tau_{c}=\frac{1}{2}-y_{c}^{2} .
$$

Likewise, for any fixed point that approaches the singularity $z=1$, it follows from (33) that merger occurs at the insert strength: 


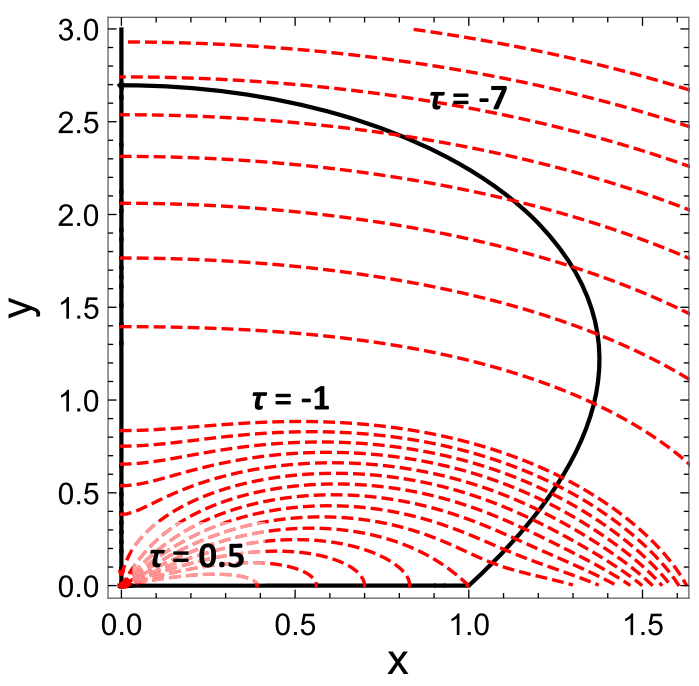

FIG. 11. Contours of the real (red dashed) and imaginary (solid black) parts of the complex function in (33), illustrating the locations of fixed points in one quadrant of the transverse plane for all values of $\tau$. The origin is always a fixed point. The remaining fixed points move over the solid black curves as $\tau$ varies. Dashed red curves are shown for decreasing values of $\tau$ ranging from $\tau=0.5$ to $\tau=-1$ (in decrements of 0.1 ) and from $\tau=-1$ to $\tau=-9$ (in decrements of 1 ).

$$
\tau=\lim _{z \rightarrow 1}\left(\frac{\bar{z}}{F^{\prime}(z)}\right)=0
$$

since $F^{\prime}(z)$ diverges at $z=1$. (The same result holds for $z=-1$.)

These fixed point bifurcations can be seen in Fig. 12, which illustrates the coordinates $(x, y)$ of each fixed point as a function of $\tau$. The solid lines indicate the coordinates of those fixed points with either $x$ or $y$ vanishing, while the dashed lines indicate those fixed points with both $x$ and $y$ nonzero. The stability type is indicated by color (blackstable, red-unstable). Moving from right to left ( $\tau$ decreasing), one begins with a single fixed point at the origin. At $\tau=1 / 2$, two additional fixed points are created, which move along the $x$-axis until colliding with the singular points at $\tau=0$. At $\tau=0$, each of these fixed points splits into two, resulting in four fixed points (in addition to the fixed point at the origin). Two additional fixed points emerge from the origin at $\tau=-1 / 2$. The latter two fixed points move along the $y$-axis until merging with those located on the dashed curve at $\tau=\tau_{c}$. Outside the range $\tau_{c} \leq \tau \leq 1 / 2$, no additional fixed point bifurcations occur.

There also exist local minima of $V$ that lie along the branch cuts (Fig. 1) for certain values of $\tau$. At these points, $\partial_{x} V=0$, while $\partial_{y} V$ is not defined (as it jumps discontinuously across the branch cut). We refer to these points as pseudofixed points. At each of these points,

$$
y=0, \quad|x|>1, \quad \partial_{x} V=0 .
$$
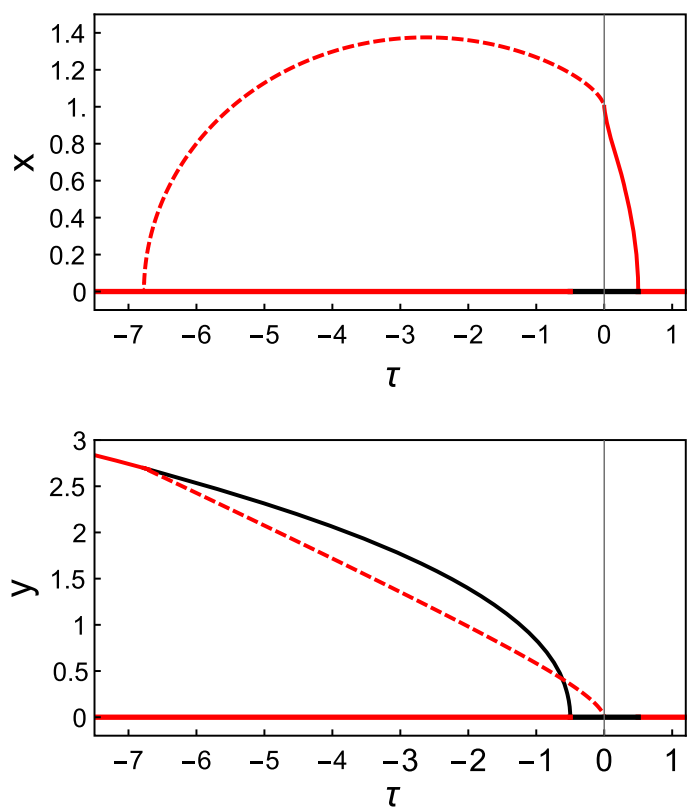

FIG. 12. Spatial coordinates $(x, y)$ of the dynamical fixed points lying in one quadrant of the transverse plane are shown as functions of the parameter $\tau$, illustrating the fixed point bifurcations discussed in Sec. VI A. Each figure must be extended by symmetry to $x<0$ or $y<0$. (Black) Stable fixed point. (Red) Unstable fixed point. Compare the summary in Table II.

Note that for $x>1$,

$$
\partial_{x} V(x, 0)=x+\frac{\tau x}{x^{2}-1}-\frac{\tau \operatorname{arccosh} x}{\left(x^{2}-1\right)^{3 / 2}} .
$$

Pseudofixed points occur at those values of $x$ where (39) vanishes. Equation (39) has one root $x>1$ when $\tau<-3 / 2$. This pseudofixed point may merge with the singularity $(1,0)$. To see this, note that a finite one-sided limit exists as the singular point is approached along the line $y=0, x>1$, since:

$$
\lim _{x \rightarrow 1+} \partial_{x} V(x, 0)=1+\frac{2 \tau}{3} .
$$

Note that (40) vanishes when $\tau=-3 / 2$. Thus, the pseudofixed point emerges from the singularity at $\tau=-3 / 2$ and moves toward increasing $|x|$ as $\tau$ decreases.

The complete set of values $\tau$ at which fixed point bifurcations occur is therefore given by:

$$
\tau \in\left\{1 / 2,0,-1 / 2,-3 / 2, \tau_{c}\right\},
$$

where $\tau_{c}$ is the value given by (36). Table II summarizes the number and type of fixed points present as $\tau$ is varied. Note that the origin is only stable in the range $-1 / 2<\tau<1 / 2$.

\section{B. Extended bifurcation diagram}

The bifurcation diagram $\Sigma$ (defined in Sec. III. A) itself undergoes topological changes as the insert strength 
TABLE II. Number and stability of fixed points (PF denotes pseudofixed point).

\begin{tabular}{llc}
\hline \hline Parameter & Fixed points & Origin stable? \\
\hline$(\tau>1 / 2)$ & 1 unstable & $\mathrm{N}$ \\
$(0<\tau<1 / 2)$ & 1 stable, 2 unstable & $\mathrm{Y}$ \\
$(-1 / 2<\tau<0)$ & 1 stable, 4 unstable & $\mathrm{Y}$ \\
$(-3 / 2<\tau<-1 / 2)$ & 2 stable, 5 unstable & $\mathrm{N}$ \\
$\left(\tau_{c}<\tau<-3 / 2\right)$ & 2 stable, 5 unstable, 2 PF & $\mathrm{N}$ \\
$\left(\tau<\tau_{c}\right)$ & 3 unstable, 2 PF & $\mathrm{N}$ \\
\hline \hline
\end{tabular}

parameter $\tau$ is varied. The $\tau$-dependence of $\Sigma$ can be studied systematically by working in an extended phase space with one additional degree of freedom, as described in Appendix E. This procedure results in an extended bifurcation diagram $\Sigma^{3}$ in the $3 \mathrm{D}$ parameter space $(H, I, \tau)$. Because the structure of $\Sigma^{3}$ is difficult to visualize, we consider its intersection with several planes of the form $\tau=\tau_{0}$. Such an intersection yields the bifurcation diagram $\Sigma$ for the value $\tau=\tau_{0}$. We consider a set of values $\tau_{0}$ that is sufficient to demonstrate the range of topologically distinct behaviors for $\Sigma$.

The extended bifurcation diagram $\Sigma^{3}$ divides the parameter space $(H, I, \tau)$ into 8 distinct regions, separating a total of 7 distinct types of regular level sets of $\mathcal{F}$, which will be denoted by the letter codes A-G. Four of these types were shown in Fig. 4. The remaining three are shown in Fig. 13,
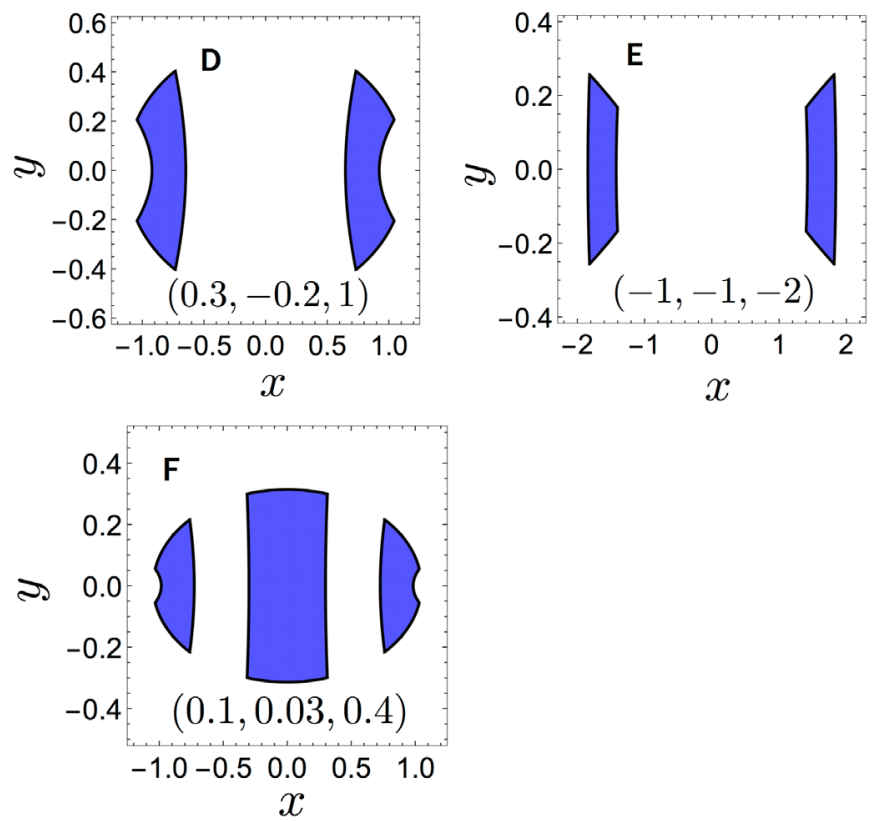

FIG. 13. Projections of three additional level set types, completing the collection (A-G) begun in Fig. 4. In each figure, the numerical triple denotes $(H, I, \tau)$. A nonresonant orbit on such a level set densely fills one of its connected components. (D) Two connected components, not intersecting the branch cuts. (E) Two connected components, intersecting the branch cuts.

(F) Three connected components. via their projections into the $(x, y)$ plane. Note that a regular level set may have $0,1,2,3$, or 4 connected components, and that all such level sets are bounded in the spatial variables $(x, y)$.

For each $\tau \in \mathbb{R}, \Sigma$ is defined by the four curves given in (17) and their intersections, where the values $\left(\xi_{\min }, \eta_{\min }\right.$, $\left.s_{\min }, t_{\min }\right)$ are defined piecewise for all $\tau \in \mathbb{R}$ as follows:

$$
\begin{gathered}
\xi_{\min }= \begin{cases}1, & \tau \geq-3 / 2, \\
\xi>1 \text { zero } I_{A}(\xi)-2 H_{A}(\xi), & \tau<-3 / 2\end{cases} \\
\eta_{\min }= \begin{cases}0, & \tau \geq 1 / 2, \\
\eta>0 \text { zero } I_{B}(\eta)-2 H_{B}(\eta), & 0<\tau<1 / 2, \\
\text { solution } H_{A}=H_{B}, I_{A}=I_{B}, & \tau_{c}<\tau<0, \\
0, & \tau<\tau_{c}\end{cases} \\
s_{\min }= \begin{cases}-\infty, & \tau>0, \\
0, & -1 / 2 \leq \tau \leq 0, \\
1 / 2+\tau, & -3 / 2 \leq \tau<-1 / 2, \\
H_{A}\left(\xi_{\min }\right), & \tau<-3 / 2,\end{cases} \\
t_{\min }= \begin{cases}0, & \tau \geq-1 / 2, \\
H_{A}\left(\xi_{d}\right), \xi_{d}>1 \text { zero } I_{A}(\xi)=0, & \tau<-1 / 2,\end{cases}
\end{gathered}
$$

Figures 14-19 illustrate the bifurcation diagram $\Sigma$ obtained using 7 representative values of $\tau$. Information about the classification of critical points is indicated using
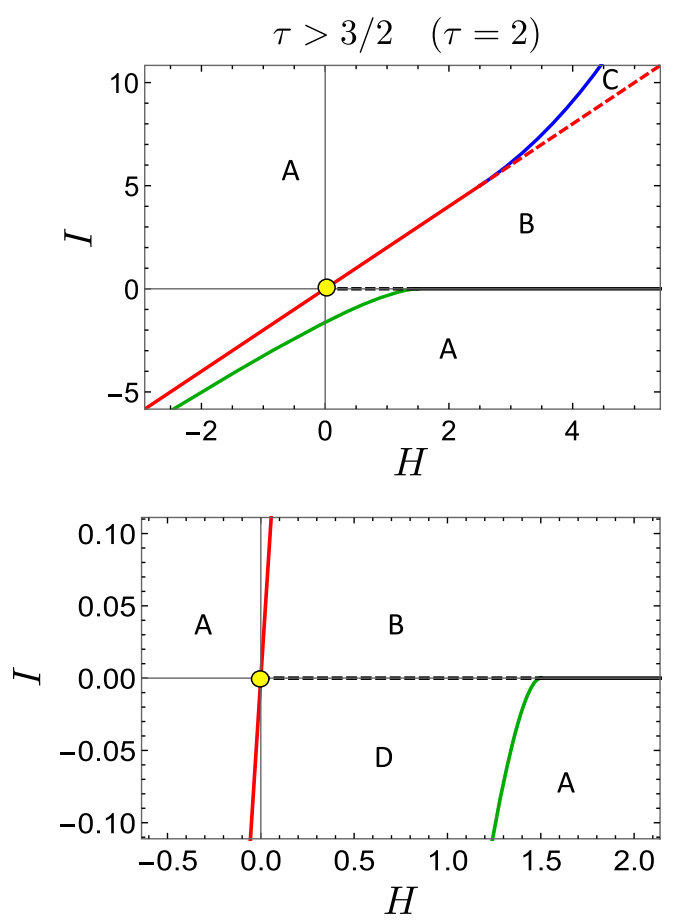

FIG. 14. Bifurcation diagram $\Sigma$ for the insert strength value $\tau=2$, dividing the $(H, I)$ plane into five distinct regions. (Upper) Default view, showing all available regions. (Lower) Scaled-up view near $(H, I)=(0,0)$. 

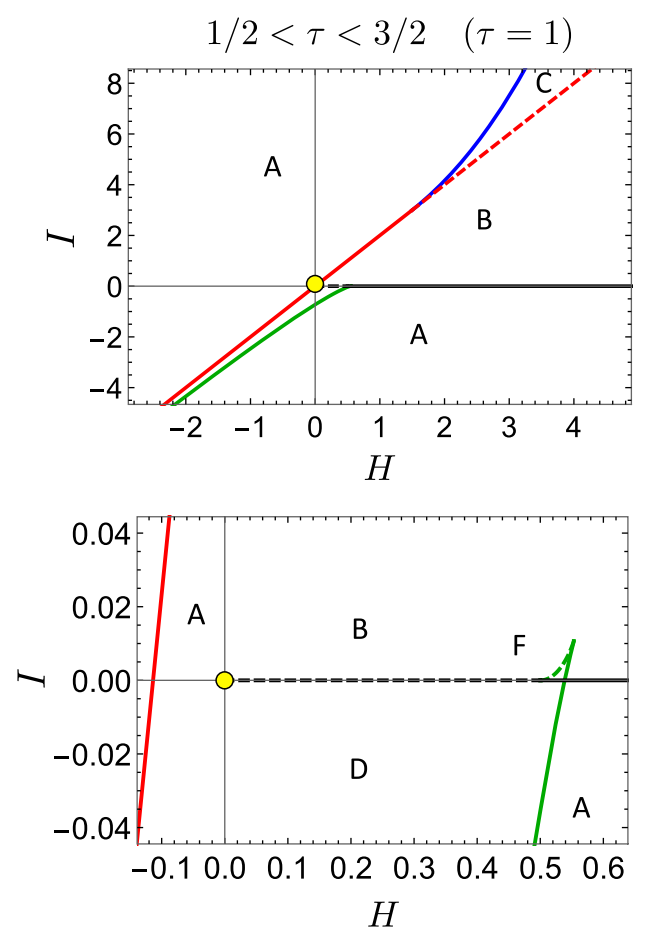

FIG. 15. Bifurcation diagram $\Sigma$ for the insert strength value $\tau=1$, dividing the $(H, I)$ plane into six distinct regions. (Upper) Default view. (Lower) Scaled-up view near $(H, I)=(0,0)$, in which the region of type $\mathrm{F}$ is visible.
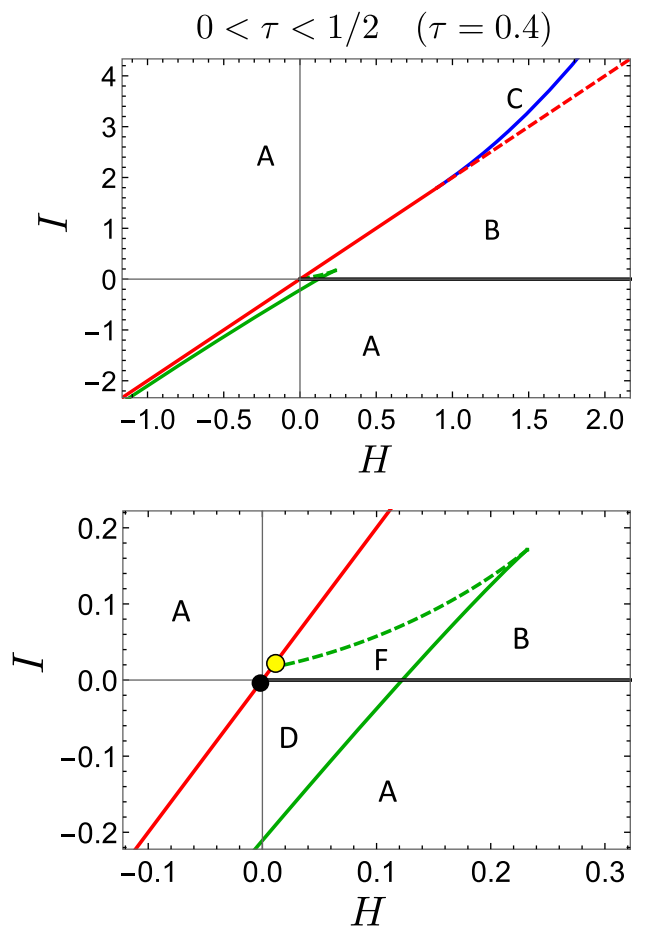

FIG. 16. Bifurcation diagram $\Sigma$ for the insert strength value $\tau=0.4$, dividing the $(H, I)$ plane into six distinct regions. (Upper) Default view. (Lower) Scaled-up view near $(H, I)=(0,0)$, in which the region of type $\mathrm{F}$ is visible.
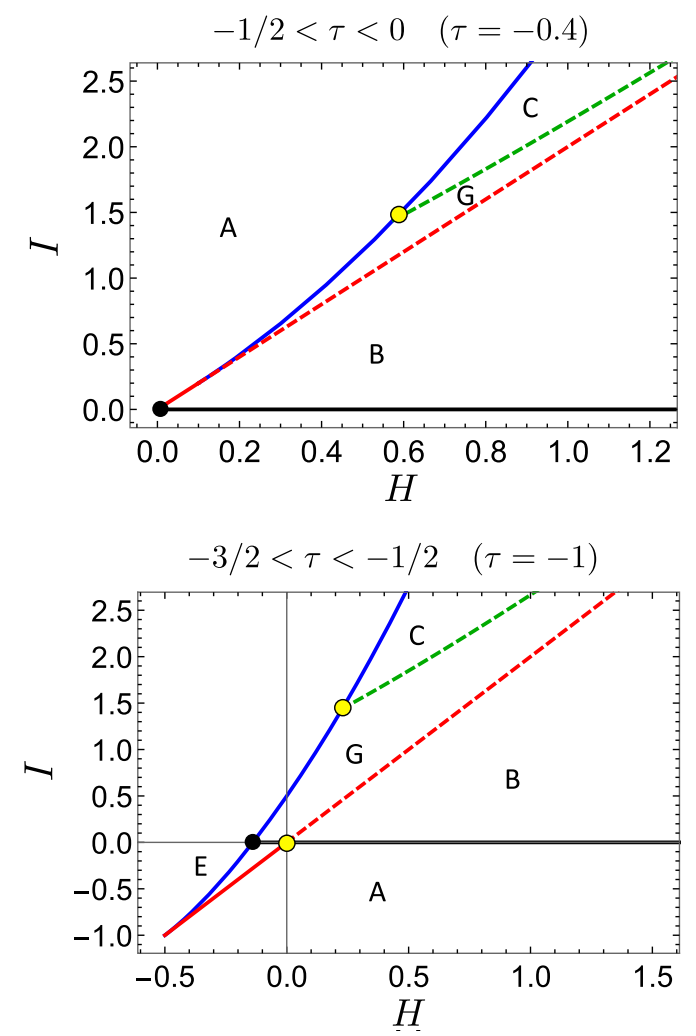

FIG. 17. (Upper) Bifurcation diagram $\Sigma$ for the insert strength value $\tau=-0.4$, dividing the $(H, I)$ plane into four distinct regions. (Lower) Bifurcation diagram $\Sigma$ for the insert strength parameter $\tau=-1$, dividing the $(H, I)$ plane into five distinct regions. The cusp at $(-1 / 2,-1)$ corresponds to the pair of singular points, excluded from the phase space $M$.

the scheme described in Sec. IV C. In several of these figures, a second illustration of the bifurcation diagram is shown at a larger scale, in order to highlight features that are difficult to resolve by eye. Each fixed point bifurcation, as described in the previous section, results in a topological change in the bifurcation diagram $\Sigma$. One additional topological change of $\Sigma$, which is related to the periodic orbits instead of the fixed points, occurs at $\tau=3 / 2$.

When $\tau>0$, the singularities at $( \pm 1,0)$ are attractive, and the two values $H$ and $I$ are not bounded below. In particular, for $\tau>3 / 2$, we see from Table II that there is one unstable fixed point at the origin, which is reflected by the yellow dot in Fig. 14. As $\tau$ decreases through $\tau=3 / 2$, a new region appears with level sets of type F (Fig. 15), and a new family of unstable periodic orbits appears (dashed green). A fixed point bifurcation occurs at $\tau=1 / 2$, and the region of type F intersects the line $I=2 H$ (red curve). The diagram changes suddenly when $\tau<0$, as $H$ and $I$ are now bounded below, and the regions of type $\mathrm{D}$ and $\mathrm{F}$ disappear, replaced by a new region of type G. At $\tau=-1 / 2$, the fixed point bifurcation results in a new region of type $E$, containing values with $H<0$ and $I<0$. Once $\tau$ decreases through the value $-3 / 2$, the cusp at the lowermost values of $(H, I)$ transitions to a level set containing the pseudofixed 


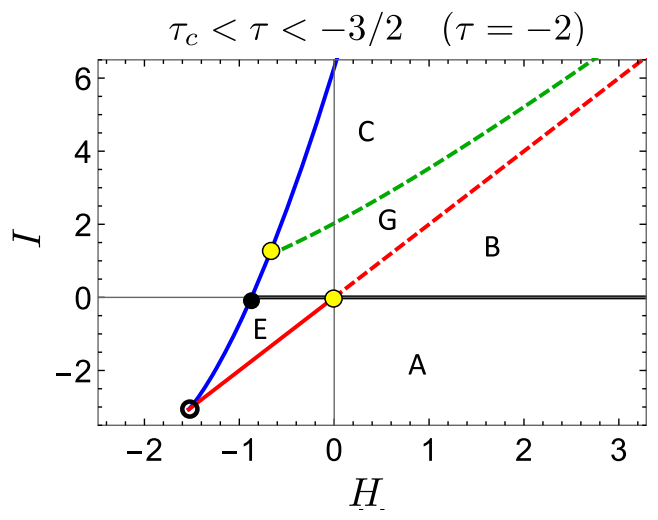

FIG. 18. Bifurcation diagram $\Sigma$ for the insert strength value $\tau=-2$, dividing the $(H, I)$ plane into five distinct regions. The open dot at $(-3 / 2,-3)$ denotes the level set containing the pair of pseudo-fixed points, described in Sec. VI A.

point. At $\tau=\tau_{c}$, a final fixed point bifurcation occurs, and a new family of unstable periodic orbits appears (on the boundary between regions $\mathrm{C}$ and $\mathrm{E}$ ). No additional topological changes occur outside the range $\tau_{c} \leq \tau \leq 3 / 2$.

\section{Critical initial conditions}

In this subsection, we comment on the $\tau$-dependence of the set of critical initial conditions $\mathcal{F}^{-1}(\Sigma)$ defined in
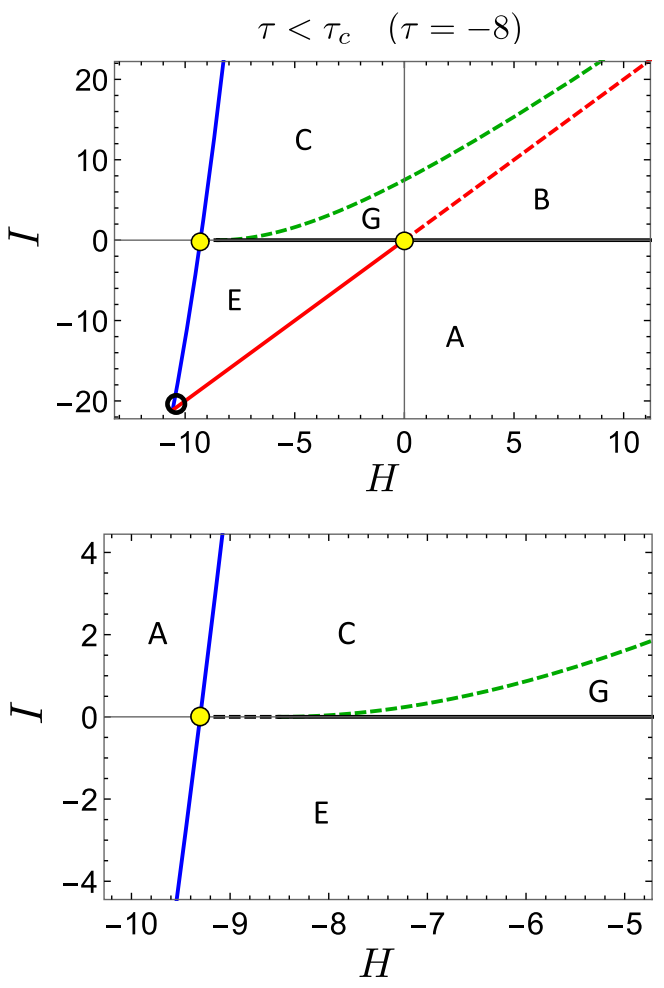

FIG. 19. Bifurcation diagram $\Sigma$ for the insert strength value $\tau=-8$, dividing the $(H, I)$ plane into five distinct regions. (Upper) Default view. The open dot has the same meaning as in Fig. 18. (Lower) Scaled-up view showing clearly the stability of boundaries between regions of type $\mathrm{C}, \mathrm{G}$, and $\mathrm{E}$.
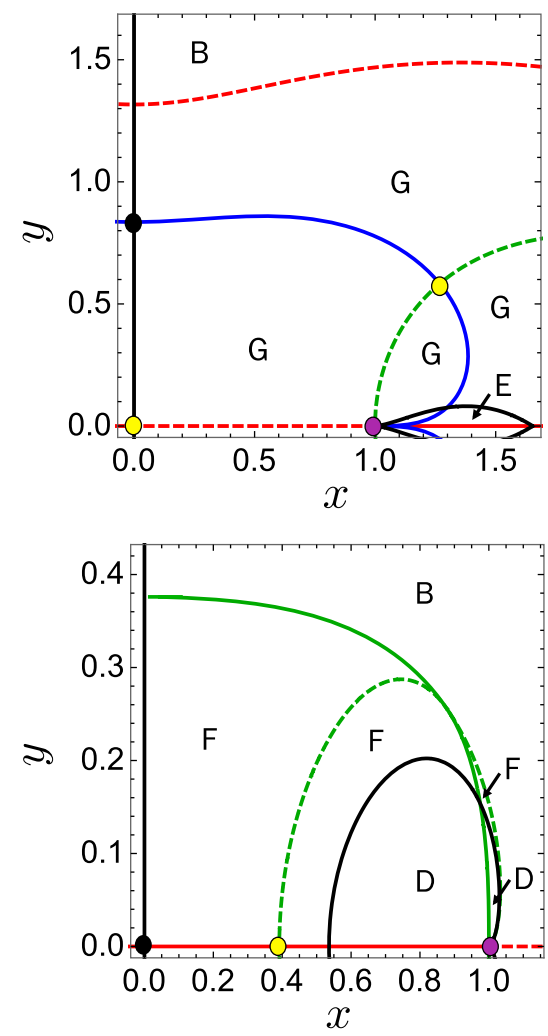

FIG. 20. Critical initial condition set $\mathcal{F}^{-1}(\Sigma)$, shown in the $(x, y)$ plane with $p_{x}=p_{y}=0$, for insert strengths $\tau=-1$ (upper) and $\tau=+0.4$ (lower). Only one quadrant is shown. Colors and labels correspond to those found in Figs. 16-17. Black dot-stable fixed point. Yellow dot—unstable fixed point. Purple dot-singular point (not considered part of the phase space $M)$.

Sec. V. The set of critical initial conditions $(x, y)$ with $p_{x}=$ $p_{y}=0$ is shown in Fig. 20 for the two values $\tau=-1$ and $\tau=0.4$, showing one case with an unstable origin, and one case with reversed insert sign (attractive singularities). (Compare Fig. 6.) Rather than providing similar figures for all 7 cases shown in Figs. 14-19, we note that many features of $\mathcal{F}^{-1}(\Sigma)$ are reflected in the corresponding bifurcation diagram $\Sigma$. For this purpose, it is helpful to note that all points with $p_{x}=p_{y}=0$ on the horizontal axis map under $\mathcal{F}$ to the curve (17c) (shown in red), and all points on the vertical axis map under $\mathcal{F}$ to the black curve (17d) (shown in black).

For a beam on-axis in the nonlinear magnet, the dynamics of primary interest is motion about a stable fixed point at the origin. Recall that the origin is stable for $-1 / 2<\tau<1 / 2$. In this case, one is interested in those critical structures in the phase space that are nearest the origin. Write $\Sigma=\Sigma_{s} \cup \Sigma_{u} \cup \Sigma_{d}$, where $\Sigma_{s}\left(\Sigma_{u}, \Sigma_{d}\right)$ denotes the image under $\mathcal{F}$ of all stable (unstable, degenerate) critical points in $K$. We then define the distance (within the plane $p_{x}=p_{y}=0$ ) to the innermost unstable critical structure by: 


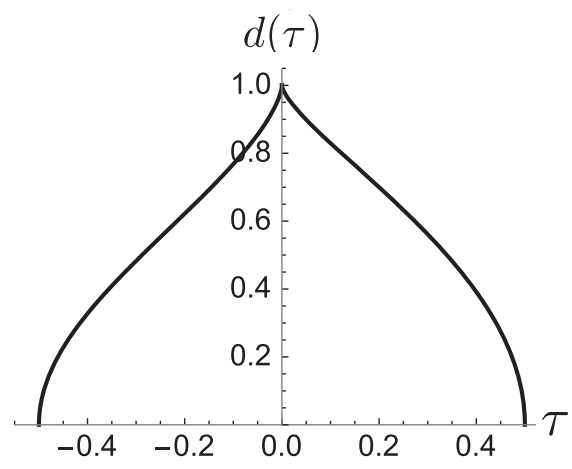

FIG. 21. Distance (42) from the origin to the innermost unstable critical structure in the plane $p_{x}=p_{y}=0$ as a function of $\tau$. The origin itself is unstable when $|\tau|>1 / 2$. When $\tau=0$, the dynamical system described by (9) is linear and stable, with $d(\tau)=\infty$.

$$
d(\tau)=\inf \left\{\sqrt{x^{2}+y^{2}}:(x, 0, y, 0) \in \mathcal{F}^{-1}\left(\Sigma_{u}\right)\right\} .
$$

When $-1 / 2<\tau<0$, it is apparent from Fig. 6 that the innermost unstable structure occurs on the $x$-axis, where the red curve transitions from solid (stable) to dashed (unstable). When $0<\tau<1 / 2$, it is apparent from Fig. 20 that the innermost unstable structure occurs at the location of the unstable fixed point (yellow) on the $x$-axis. Figure 21 illustrates (42) as a function of $\tau$, showing how this distance shrinks as the magnetic insert strength is increased.

\section{CONCLUSIONS}

Any accelerator system designed to a strongly nonlinear working point requires theoretical tools that are sufficient for understanding the nominal single-particle dynamics. For rings based on nonlinear integrable optics [1,2], tools from the geometric theory of integrable Hamiltonian systems may be applied [9-11] that do not require the use of special coordinates or action-angle variables, and which extend to any number of degrees of freedom. In particular, the geometry of orbits is clarified by studying how the phase space is partitioned into connected components of the level sets of the invariants of motion (the Liouville foliation). The critical level sets contain all of the phase space structures of qualitative dynamical interest, including the fixed points, critical periodic orbits, and separatrixlike structures. We have described how these may be determined and visualized using the bifurcation diagram $\Sigma$. Key results are summarized as follows:

(i) The single-particle nominal on-energy dynamics in IOTA is described by the integrable Hamiltonian (9)-(10) with second invariant (11).

(ii) A global analysis of the orbits is achieved by studying the momentum mapping (15) (its level sets, critical points, and critical values). This begins with a search for its critical points (16). (iii) The bifurcation diagram (Fig. 3) contains critical values of the momentum mapping, where the geometry of the level sets (and hence, the geometry of the orbits) changes qualitatively.

(iv) The critical points include dynamical fixed points and critical periodic orbits, and these may be classified in a way that reflects the local stability of motion (Table I).

(v) Level sets corresponding to critical values (critical level sets) partition the phase space into regions with distinct dynamical behavior (Figs. 6-7).

(vi) Analysis of the fixed point condition (32) can be used to study the number and stability of fixed points as the nonlinear insert strength $\tau$ is varied [(12) and Table II].

(vii) A global picture of the dynamics for all values of $\tau$ is obtained by extending the bifurcation diagram to the 3D parameter space $(H, I, \tau)$. Figs. (14-19).

The application of these methods to the IOTA ring reveals a rich diversity of accessible dynamical behavior that could be explored experimentally, subject to the limitations of the physical aperture. Understanding the dynamical dependence on the system parameters $(H, I, \tau)$ may also indicate new machine operating points and help to guide future accelerator designs [12] based on similar nonlinear magnetic elements. While we have not addressed the computation of characteristic orbital frequencies in this paper, we remark that this information may be obtained from the momentum mapping $\mathcal{F}$, by evaluating a set of appropriately defined integrals over paths that lie within the level sets of $\mathcal{F}$. This is a topic of ongoing research.

\section{ACKNOWLEDGMENTS}

The authors thank A. Valishev and the IOTA collaboration teams at Fermilab and RadiaSoft for discussions. This work was supported by the Director, Office of Science of the U.S. Department of Energy under Contract No. DEAC02-05CH11231, and made use of computer resources at the National Energy Research Scientific Computing Center. The authors acknowledge support from the U.S. DOE Early Career Research Program under the Office of High Energy Physics.

\section{APPENDIX A: SPECIAL FAMILIES OF CRITICAL POINTS}

Expressing the forms $d H$ and $d I$ in the local phase space coordinates $\left(\zeta_{1}, \zeta_{2}, \zeta_{3}, \zeta_{4}\right)=\left(x, p_{x}, y, p_{y}\right)$ gives:

$$
d H=\sum_{j=1}^{4}\left(\frac{\partial H}{\partial \zeta_{j}}\right) d \zeta_{j}, \quad d I=\sum_{k=1}^{4}\left(\frac{\partial I}{\partial \zeta_{k}}\right) d \zeta_{k} .
$$

Taking the wedge product gives, after using the antisymmetry of $\wedge$ : 
$d H \wedge d I=\sum_{k=2}^{4} \sum_{j=1}^{k-1}\left(\frac{\partial H}{\partial \zeta_{j}} \frac{\partial I}{\partial \zeta_{k}}-\frac{\partial H}{\partial \zeta_{k}} \frac{\partial I}{\partial \zeta_{j}}\right) d \zeta_{j} \wedge d \zeta_{k}$.

The critical points of $\mathcal{F}$ occur where (A2) vanishes, which occurs if and only if each of the six coefficients in parentheses in (A2) vanishes. Rather than solving this system of 6 equations in 4 unknowns directly, it is simplest to search for solutions of (A2) satisfying the following special conditions (reducing the problem from four to two unknowns).

(1) Critical points located in the plane $p_{x}=p_{y}=0$ :

This is the set of all points $(x, y)$ satisfying:

$\left(x-\tau \partial_{x} U\right)\left(\tau \partial_{y} W\right)+\left(y-\tau \partial_{y} U\right)\left(2 x-\tau \partial_{x} W\right)=0$,

where $U$ and $W$ are the functions appearing in (10)-(11). This set includes, in particular, all of the dynamical fixed points.

(2) Critical points located in the plane $x=y=0$ :

This is the set of all points $\left(p_{x}, p_{y}\right)$ with either $p_{x}=0$ or $p_{y}=0$.

(3) Critical points located in the plane $x=p_{x}=0$ :

All points in this plane are critical points.

(4) Critical points located in the plane $y=p_{y}=0$ :

All points in this plane are critical points.

(5) Critical points located in the plane $y=p_{x}=0$ :

This is the set of all points $\left(x, p_{y}\right)$ with either $p_{y}=0$ or

$$
x p_{y}^{2}-\left(x-\tau \partial_{x} U\right)\left(x^{2}-1\right)=0,
$$

where $U$ is the function appearing in (10).

(6) Critical points located in the plane $x=p_{y}=0$ :

This is the set of all points $\left(p_{x}, y\right)$ with either $p_{x}=0$ or

$$
y p_{x}^{2}-\left(y-\tau \partial_{y} U\right)\left(y^{2}+1\right)=0,
$$

where $U$ is the function appearing in (10).

Next, note that the Hamiltonian flow maps critical points to critical points. To see this, let $p \in K$ be a critical point, and let $\phi_{t}(t \in \mathbb{R})$ denote the Hamiltonian flow. The fact that $f_{1}, \ldots, f_{n}$ are invariant under $\phi_{t}$ implies that $\mathcal{F} \circ \phi_{t}=\mathcal{F}$. Applying the chain rule to the Jacobian at $p$ gives:

$$
D \mathcal{F}_{p}=D\left(\mathcal{F} \circ \phi_{t}\right)_{p}=D \mathcal{F}_{\phi_{t}(p)} D\left(\phi_{t}\right)_{p} .
$$

Since $D\left(\phi_{t}\right)_{p}$ is a symplectic matrix, it is invertible. Thus (A6) implies,

$$
\operatorname{rank}\left(D \mathcal{F}_{\phi_{t}(p)}\right)=\operatorname{rank}\left(D \mathcal{F}_{p}\right) .
$$

Since $p$ satisfies (14), so does $\phi_{t}(p)$, and therefore $\phi_{t}(p) \in K$.

Thus, the orbits of the points defined by (1)-(6) consist entirely of critical points. It follows from the structure of the level sets (Appendix B) and their symmetry under $x \mapsto-x$ and $y \mapsto-y$ that the orbit of every critical point must intersect at least one of these six planes, and this exhausts the set $K$ of critical points.

\section{APPENDIX B: LEVEL SETS}

We wish to visualize the level set $\mathcal{F}^{-1}\left(\left\{\left(H_{0}, I_{0}\right)\right\}\right)$ in the 4D phase space $M$, corresponding to invariant values $H=H_{0}$ and $I=I_{0}$. This can be done through intersection with a 2D surface to yield a Poincaré section, as described in Sec. IV B, or by projection into one or more planes. Below, we consider projection into the $(x, y)$ plane, which also yields a convenient 2D parametrization of the level surface.

Solving for the magnitude of momentum in the equation $H=H_{0}$ gives:

$$
|p|=\sqrt{2\left[H_{0}-V(x, y)\right]}, \quad V(x, y) \leq H_{0} .
$$

A real solution of (B1) exists for all values of $(x, y)$ satisfying the inequality. Define

$$
\left(p_{x}, p_{y}\right)=(|p| \cos \phi,|p| \sin \phi), \quad t=e^{2 i \phi} .
$$

The equation $I=I_{0}$ then takes the form of an equation for $t$ :

$$
A(x, y) t^{2}+B(x, y) t+C(x, y)=0,
$$

where

$$
A(x, y)=\bar{C}(x, y)=\frac{|p|^{2}}{4}\left(1-x^{2}+y^{2}+2 i x y\right),
$$

$B(x, y)=\frac{|p|^{2}}{2}\left(1+x^{2}+y^{2}\right)+x^{2}-\tau W(x, y)-I_{0}$,

and $|p|$ is given by (B1). A solution of (B3) for $t$ exists on the unit circle for all values of $(x, y)$ satisfying:

$$
B(x, y)^{2}-4|A(x, y)|^{2} \leq 0 .
$$

Using such $t$ in (B1)-(B2) gives the value(s) of $\left(p_{x}, p_{y}\right)$ that lie on the desired level surface over the point $(x, y)$.

The projection of the level set into the $(x, y)$ plane is therefore given by the set of points satisfying simultaneously the two inequalities in (B1) and (B6). It is convenient that (B6) factors when expressed in elliptic coordinates to give:

$$
F_{1}(\xi) F_{2}(\eta) \leq 0
$$

where the coordinates $(\xi, \eta)$ are related to $(x, y)$ by:

$$
(x, y)=\left(\xi \eta, \pm \sqrt{\left(\xi^{2}-1\right)\left(1-\eta^{2}\right)}\right)
$$

and the two functions $F_{1}$ and $F_{2}$ are: 
$F_{1}(\xi)=I_{0}+\xi^{2}\left(-1-2 H_{0}+\xi^{2}\right)+2 \tau \xi \sqrt{\xi^{2}-1} \operatorname{arccosh}(\xi)$,

$F_{2}(\eta)=I_{0}+\eta^{2}\left(-1-2 H_{0}+\eta^{2}\right)+2 \tau \eta \sqrt{1-\eta^{2}} \arcsin (\eta)$.

Points on the boundary of the level set in the $(x, y)$ plane occur only where equality holds in (B7), so that

$$
F_{1}(\xi)=0 \quad \text { or } \quad F_{2}(\eta)=0 .
$$

Solution of (B9) yields an ellipse $\left(\xi=\xi_{0}\right)$ or hyperbola $\left(\eta=\eta_{0}\right)$ in the $(x, y)$ plane, with foci located at the two singular points $(x, y)=( \pm 1,0)$. The boundary of the projected level set then consists of one or more segments of these ellipses and hyperbolae, as constrained by the additional inequality (B1).

\section{APPENDIX C: BIFURCATION DIAGRAM}

The set $K$ of critical points satisfying (16) must generally be obtained by searching numerically for the simultaneous zeros of a set of real-valued functions on the phase space, as in Appendix A. Evaluating the invariants at the locations of these numerically determined critical points then yields the bifurcation diagram $\Sigma=\mathcal{F}(K)$.

In the special case of the Hamiltonian (9)-(10), one may also use the results of Appendix B to obtain an explicit parametrization of the bifurcation diagram as follows. The boundary of each level set's projection into the $(x, y)$ plane is determined by the zeros of the two functions $F_{1}$ and $F_{2}$ (B9). The topology of the level set therefore changes at each value of $\left(H_{0}, I_{0}\right)$ that coincides with a change in the number of zeros of $F_{1}$ or $F_{2}$. This coincides with the presence of a double root, where $F_{1}=F_{1}^{\prime}=0$ or $F_{2}=F_{2}^{\prime}=0$. Also, the boundary ellipse $\left(\xi=\xi_{0}\right)$ or hyperbola $\left(\eta=\eta_{0}\right)$ becomes degenerate at the limiting values $\xi_{0}=1, \eta_{0}=0$, or $\eta_{0}=1$. The topology of the level set may change at any value of $\left(H_{0}, I_{0}\right)$ for which $F_{1}$ or $F_{2}$ has a root at one of these values. One then obtains the parametrization (17) as follows:

(A) Solve $F_{1}^{\prime}=0$ for $H_{0}$, to obtain the parametrization of $H_{A}$. Substitute $H_{0}=H_{A}$ into $F_{1}=0$, and solve for $I_{0}$ to obtain the parametrization of $I_{A}$.

(B) Solve $F_{2}^{\prime}=0$ for $H_{0}$, to obtain the parametrization of $H_{B}$. Substitute $H_{0}=H_{B}$ into $F_{2}=0$, and solve for $I_{0}$ to obtain the parametrization of $I_{B}$.

(C) Note that:

$$
\lim _{\xi \rightarrow 1} F_{1}(\xi)=\lim _{\eta \rightarrow 1} F_{2}(\eta)=-2 H_{0}+I_{0} .
$$

Setting $(\mathrm{C} 1)$ to zero yields $I_{0}=2 H_{0}$, giving $\left(H_{C}, I_{C}\right)$. (D) Note that:

$$
\lim _{\eta \rightarrow 0} F_{2}(\eta)=I_{0} .
$$

Setting (C2) to zero yields $I_{0}=0$, giving $\left(H_{D}, I_{D}\right)$. Finally, the values $\left(\xi_{\min }, \eta_{\min }, s_{\min }, t_{\min }\right)$ are defined by the intersection points of the four curves (17a)-(17d).
This procedure yields the same results as a direct evaluation of $\Sigma=\mathcal{F}(K)$ based on the results of Appendix A. In particular, taking the images of the 6 families of critical points in Appendix A gives the following result:

(1) In the plane $p_{x}=p_{y}=0$, solving (A3) numerically for $(x, y)$ and evaluating $(H, I)$ at these points yields critical values lying on the four curves (A)-(D) of (17). This was verified numerically for a range of $\tau$, including $\tau=-0.4$.

(2) In the plane $x=y=0$, the case $p_{x}=0$ gives by direct evaluation that $I=0$ (on curve D). Similarly, the case $p_{y}=0$ gives $I=2 H$ (on curve C).

(3) In the plane $x=p_{x}=0$, direct evaluation gives that $I=0$ (on curve $\mathrm{D})$.

(4) In the plane $y=p_{y}=0$, direct evaluation gives that $I=2 H$ (on curve C).

(5) In the plane $y=p_{x}=0$, the case $p_{y}=0$ gives $I=$ $2 H$ (on curve C). Otherwise, solve (A4) for $p_{y}^{2}$ and substitute into $(H, I)$. If $|x|>1$, taking $x=\xi$ gives the curve $\left(H_{A}, I_{A}\right)$. If $|x|<1$, taking $x=\eta$ gives the curve $\left(H_{B}, I_{B}\right)$.

(6) In the plane $x=p_{y}=0$, the case $p_{x}=0$ gives $I=0$ (curve D). Otherwise, solve (A5) for $p_{x}^{2}$ and substitute into $(H, I)$. Taking $y= \pm \sqrt{\xi^{2}-1}$ gives the curve $\left(H_{A}, I_{A}\right)$.

It follows that all critical points have invariant values $(H, I)$ that lie on the four curves defined in (17).

\section{APPENDIX D: DYNAMICAL STABILITY}

A general Hamiltonian flow on a phase space of dimension $2 n$ is described by the equations of motion:

$$
\dot{\zeta}=J \nabla H(\zeta)
$$

where $\zeta=\left(q_{1}, p_{1}, \ldots, q_{n}, p_{n}\right)$, and $J$ denotes the $2 n \times 2 n$ matrix of the symplectic form:

$$
J=\operatorname{diag}\left(J_{1}, \ldots, J_{1}\right), \quad J_{1}=\left(\begin{array}{cc}
0 & 1 \\
-1 & 0
\end{array}\right) .
$$

Let $\zeta_{d}$ denote an orbit of (D1) with period $T \geq 0$. (In this Appendix, we consider both periodic orbits and fixed points, where for a fixed point we set $T=0$.) Dynamical stability is determined by linearizing (D1) about the orbit $\zeta_{d}$, yielding the variational equations:

$$
\delta \dot{\zeta}=J S(t) \delta \zeta, \quad S(t)=\operatorname{Hess}_{\zeta_{d}(t)}(H) .
$$

The solution of (D3) takes the form $\delta \zeta(t)=R(t) \delta \zeta(0)$, where the linear transport matrix $R$ satisfies the matrix equation [20]:

$$
\dot{R}=J S(t) R, \quad R(0)=I d,
$$

and $I d$ denotes the $2 n \times 2 n$ identity matrix. By the FloquetLyapunov theorem ([25], 2.5.2 and 2.1.1), there exist a real 
symplectic matrix $P(t)$ and a real symmetric matrix $B$ such that:

$$
R(t)=P(t) e^{t J B}, \quad P(0)=I d,
$$

where $P$ is periodic in $t$ with period $T$ or $2 T$. The eigenvalues of $J B$ are called the characteristic exponents of the orbit $\zeta_{d}$. The orbit $\zeta_{d}$ is said to be (spectrally) stable if $J B$ is diagonalizable and all of its eigenvalues are purely imaginary. In this case, solutions of (D3) remain bounded for all $t \in \mathbb{R}$ ([25], 5.1.1). Note that, if $\lambda \in \mathbb{C}$ is a characteristic exponent, then so are $\pm \lambda, \pm \bar{\lambda}$ ([25], 2.3.1).

At a fixed point $\zeta_{d}$, the matrix $S(t)$ is independent of $t$. It follows that we may take $B=S$ and $P=I d$ in (D5), and the stability problem is reduced to studying the eigenvalues of $J S$. For a Hamiltonian in two degrees of freedom of the form (9), the eigenvalues $\sigma$ of $J S$ are the roots of:

$$
\operatorname{det}(J S-\sigma I d)=\operatorname{det}\left(\operatorname{Hess}(V)+\sigma^{2} I d\right)=0 .
$$

If $\lambda_{1}, \lambda_{2}$ denote the eigenvalues of $\operatorname{Hess}(V)$, it follows that the 4 characteristic exponents are given by:

$$
\sigma_{j}^{( \pm)}= \pm i \lambda_{j}^{1 / 2}, \quad(j=1,2) .
$$

Since $\operatorname{Hess}(V)$ is a symmetric matrix, each $\lambda_{j} \in \mathbb{R}$. It then follows from (D7) that a fixed point of the system (9) is (spectrally) stable if and only if $\lambda_{1}, \lambda_{2}>0$.

\section{APPENDIX E: EXTENDED PHASE SPACE}

We may extend the theory of Sec. III to treat the $\tau$-dependence of the Hamiltonian (9) as follows. Let $M^{4}$ denote the usual $4 \mathrm{D}$ phase space, with its canonical coordinates $\left(x, p_{x}, y, p_{y}\right)$. We define an extended phase space $M^{6}=M^{4} \times S^{1} \times \mathbb{R}$, where $S^{1}$ denotes the unit circle, with canonical coordinates $\left(x, p_{x}, y, p_{y}, \theta, \tau\right)$. Here $\theta$ is an angle variable with $e^{i \theta} \in S^{1}$, and $\tau \in \mathbb{R}$ is the nonlinear insert strength. Define a Hamiltonian $\tilde{H}$ on the phase space $M^{6}$ using the expression (9), where $\tau$ is now a dynamical variable, and define a function $\tilde{I}$ on $M^{6}$ using the expression (11). Then $\tilde{H}, \tilde{I}$, and $\tau$ are functionally independent on $M^{6}$, and one may verify that:

$$
\{\tilde{H}, \tau\}=0, \quad\{\tilde{I}, \tau\}=0, \quad\{\tilde{H}, \tilde{I}\}=0 .
$$

This defines an integrable Hamiltonian system on the extended phase space $M^{6}$, with invariants of motion $f_{1}=\tilde{H}, f_{2}=\tilde{I}$, and $f_{3}=\tau$, to which the theory of Sec. III can be applied. In this way, we obtain an extended bifurcation diagram $\Sigma^{3}$ in the parameter space $(\tilde{H}, \tilde{I}, \tau)$. The diagram $\Sigma^{3}$ consists of four parametrized 2-surfaces and their intersections in three-dimensional space, given by the expressions in (17), where $\tau$ is now treated as a parameter. The surfaces in $\Sigma^{3}$ divide $\mathbb{R}^{3}$ into distinct regions (chambers), separating the 7 types of regular level sets shown in Figs. 4, 13. In Sec. VIB, we study the bifurcation diagram $\Sigma^{3}$ by illustrating its intersections with the planes $\tau=\tau_{0}$ (slices) at several fixed values of $\tau_{0}$.

[1] V. Danilov and S. Nagaitsev, Nonlinear accelerator lattices with one and two analytic invariants, Phys. Rev. Accel. Beams 13, 084002 (2010).

[2] S. Antipov et al., IOTA (Integrable Optics Test Accelerator): Facility and experimental beam physics program, J. Instrum. 12, T03002 (2017).

[3] E. Stern et al., Suppression of instabilities generated by an anti-damper with a nonlinear magnet element in IOTA, in Proc. IPAC'18, Vancouver, Canada (2018), THPAF068, http://accelconf.web.cern.ch/ipac2018/papers/thpaf068.pdf.

[4] S. Webb et al., Suppressing transverse beam halo with nonlinear magnetic fields, Report No. FERMILAB-PUB294-AD-APC, 2012, https://lss.fnal.gov/archive/2012/pub/ fermilab-pub-12-294-ad-apc.pdf.

[5] S. Webb et al., Effects of nonlinear decoherence on halo formation, arXiv:1205.7083v2.

[6] J. Gareyte et al., Landau damping, dynamic aperture and octupoles in LHC, CERN Technical Report Nos. LHCProject-Report-91, CERN-LHC-Project-Report-91, 1997, https://cds.cern.ch/record/321824/files/lhc-project-report-91 .pdf.

[7] C. Montag, J. Kewisch, D. Trbojevic, and F. Schmidt, Overcoming a fast transverse instability by means of octupole-induced tune spread in the Relativistic Heavy Ion Collider, Phys. Rev. Accel. Beams 5, 084401 (2002).

[8] K. G. Sonnad and J. R. Cary, Finding a nonlinear lattice with improved integrability using Lie transform perturbation theory, Phys. Rev. E 69, 056501 (2004).

[9] A. V. Bolsinov and A. T. Fomenko, Integrable Hamiltonian Systems: Geometry, Topology, Classification (Chapman \& Hall/CRC Press, Boca Raton, 2004).

[10] A. Bolsinov and A. Oshemkov, Singularities of integrable Hamiltonian systems, in Topological Methods in the Theory of Integrable Systems (Cambridge Scientific Publishing, Cambridge, England, 2006).

[11] A. Pelayo and S. Vu Ngoc, Symplectic theory of completely integrable Hamiltonian systems, Bull. Am. Math. Soc. 48, 409 (2011).

[12] J. Eldred, V. Lebedev, and A. Valishev, Rapid-cycling synchrotron for multi-megawatt proton facility at Fermilab, J. Instrum. 14, P07021 (2019).

[13] R. H. Cushman and D. A. Sadovskií, Monodromy in the hydrogen atom in crossed fields, Physica D (Amsterdam) 142, 166 (2000).

[14] N. J. Fitch, C. A. Weidner, L. P. Parazzoli, H. R. Dullin, and H. J. Lewandowski, Experimental Demonstration of Classical Hamiltonian Monodromy in the 1:1:2 Resonant Elastic Pendulum, Phys. Rev. Lett. 103, 034301 (2009).

[15] E. Assémat, K. Efstathiou, M. Joyeux, and D. Sugny, Fractional Bidromy in the Vibrational Spectrum of $\mathrm{HOCl}$, Phys. Rev. Lett. 104, 113002 (2010).

[16] I. I. Kharlamova and A. Y. Savushkin, Bifurcation diagrams involving the linear integral of Yehia, J. Phys. A: Math. Theor. 43, 105203 (2010). 
[17] C. A. Arango and G. S. Ezra, Classical Mechanics of Dipolar Asymmetric Top Molecules in Collinear Static Electric And Nonresonant Linearly Polarized Laser Fields: Energy-Momentum Diagrams, Bifurcations and Accessible Configuration Space, Int. J. Bifurcation Chaos Appl. Sci. Eng. 18, 1127-1149 (2008).

[18] F. O'Shea et al., Measurement of non-linear insert magnets, in Proceedings of the 25th Particle Accelerator Conference, PAC-2013, Pasadena, CA, 2013 (IEEE, New York, 2013), p. 922, https://accelconf.web.cern.ch/ PAC2013/papers/wepba17.pdf.

[19] C. Mitchell, Complex representation of potentials and fields for the nonlinear magnetic insert of the Integrable Optics Test Accelerator, Report No. LBNL-1007217, 2017; arXiv:1908.00036.

[20] A. J. Dragt, Lie Methods for Nonlinear Dynamics with Applications to Accelerator Physics (University of Maryland, College Park, MD, 2011), https://www.physics .umd.edu/dsat/docs/Book12Nov2019.pdf.
[21] Alternative lattice designs allow for a phase advance given by $\pi k$, for $k$ odd. The motion within the nonlinear insert is then described by the Hamiltonian (9), followed by a discontinuous phase space inversion $\zeta \mapsto-\zeta$. Several results described in this paper must be modified accordingly.

[22] V. I. Arnold, Mathematical Methods of Classical Mechanics, 2nd ed. (Springer, New York, 1989).

[23] R. Abraham and J. Marsden, Foundations of Mechanics, 2nd ed. (Addison-Wesley Publishing Co., Redwood City, CA, 1978).

[24] J. Moser and E. Zehnder, Notes on Dynamical Systems (AMS, Courant Institute of Mathematical Sciences, Providence, Rhode Island, 2005).

[25] K. R. Meyer and D. C. Offin, Introduction to Hamiltonian Dynamical Systems and the N-Body Problem, 3rd. ed. (Springer International Publishing, New York, 2017). 